\title{
Laboratory evidence for an asymmetric accretion structure upon slanted matter impact in young stars ${ }^{\star}$
}

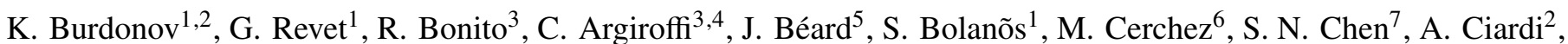
G. Espinosa ${ }^{8}$, E. Filippov ${ }^{9,10}$, S. Pikuz $^{9,11}$, R. Rodriguez ${ }^{8}$, M. Šmíd ${ }^{12}$, M. Starodubtsev ${ }^{10}$, O. Willi ${ }^{6}$, S. Orlando ${ }^{3}$, and J. Fuchs ${ }^{1}$

${ }^{1}$ LULI - CNRS, CEA, UPMC Univ Paris 06 : Sorbonne Université, Ecole Polytechnique, Institut Polytechnique de Paris, 91128 Palaiseau Cedex, France e-mail: konstantin. burdonov@polytechnique.edu

2 Sorbonne Université, Observatoire de Paris, PSL Research University, LERMA, CNRS UMR 8112, 75005 Paris, France

3 INAF, Osservatorio Astronomico di Palermo, Palermo, Italy

${ }^{4}$ Department of Physics and Chemistry, University of Palermo, 90133 Palermo, Italy

5 LNCMI, UPR 3228, CNRS-UGA-UPS-INSA, 31400 Toulouse, France

${ }^{6}$ Heinrich-Heine Universität Düsseldorf, 40225 Düsseldorf, Germany

7 ELI-NP, "Horia Hulubei” National Institute for Physics and Nuclear Engineering, 30 Reactorului Street, 077125 Bucharest-Magurele, Romania

8 Department of Physics, IUNAT, Universidad de Las Palmas de Gran Canaria, 35001 Las Palmas, Spain

9 JIHT, Russian Academy of Sciences, 125412 Moscow, Russia

10 IAP, Russian Academy of Sciences, 603950 Nizhny Novgorod, Russia

11 NRNU MEPhI, 115409 Moscow, Russia

12 Helmholtz-Zentrum Dresden-Rossendorf, 01328 Dresden, Germany

Received 17 April 2020 / Accepted 18 July 2020

\begin{abstract}
Aims. Investigating the process of matter accretion onto forming stars through scaled experiments in the laboratory is important in order to better understand star and planetary system formation and evolution. Such experiments can indeed complement observations by providing access to the processes with spatial and temporal resolution. A previous investigation revealed the existence of a twocomponent stream: a hot shell surrounding a cooler inner stream. The shell was formed by matter laterally ejected upon impact and refocused by the local magnetic field. That laboratory investigation was limited to normal incidence impacts. However, in young stellar objects, the complex structure of magnetic fields causes variability of the incident angles of the accretion columns. This led us to undertake an investigation, using laboratory plasmas, of the consequence of having a slanted accretion impacting a young star. Methods. Here, we used high power laser interactions and strong magnetic field generation in the laboratory, complemented by numerical simulations, to study the asymmetry induced upon accretion structures when columns of matter impact the surface of young stars with an oblique angle.

Results. Compared to the scenario where matter accretes perpendicularly to the star surface, we observe a strongly asymmetric plasma structure, strong lateral ejecta of matter, poor confinement of the accreted material, and reduced heating compared to the normal incidence case. Thus, slanted accretion is a configuration that seems to be capable of inducing perturbations of the chromosphere and hence possibly influencing the level of activity of the corona.
\end{abstract}

Key words. accretion, accretion disks - instabilities - magnetohydrodynamics (MHD) - shock waves - stars: pre-main sequence X-rays: stars

\section{Introduction}

The dynamics of matter accretion is highly interesting process, because of its dominant role in a wide range of astrophysical objects, including the evolution of young stars and the formation of planetary systems (e.g., Hartmann et al. 2016; Scaringi et al. 2015; Caratti o Garatti et al. 2017; Argiroffi et al. 2017). In the case of low-mass young stars, observations have provided evidence that the process of matter accretion impacts the evolution of the surrounding stellar atmosphere and, in particular, the level of coronal activity (Neuhaeuser et al. 1995; Flaccomio et al.

* Movies are available at https://www . aanda.org
2003; Stassun et al. 2004; Preibisch et al. 2005; Jardine et al. 2006; Gregory et al. 2007; Drake et al. 2009; Brickhouse et al. 2010; Orlando et al. 2011; Dupree et al. 2012; Colombo et al. 2019a). Current models also suggest possible perturbations of the stellar atmosphere by accreting streams, however, up to now there have not been many studies related to such phenomena (Orlando et al. 2010, 2013; Matsakos et al. 2013). In the solar case, perturbations of the chromosphere were observed during the impacts of cold and dense fragments previously ejected in the interplanetary medium by a solar eruption associated with a flare (Reale et al. 2013, 2014). Perturbations of the chromosphere and corona of young stars were modeled in Orlando et al. (2010, 2013). However, the levels of perturbations were not studied in detail because the models are idealized and assume only 
normal impacts. A first attempt to model oblique impacts was made by Reale et al. (2014), who described impacts of dense fragments onto the solar surface at an angle of $15^{\circ}$ with respect to normal impacts. These studies have suggested that an oblique impact may produce much stronger perturbations of the chromosphere, possible additional heating mechanism of the overlying corona, and, therefore, possible influence on the level of activity of the corona.

In our previous work (Revet et al. 2017), we modeled the perpendicular impact of an incoming collimated plasma stream onto the surface of the star, in the presence of a magnetic field co-aligned with the stream. The plasma in the experiment was shown to be scalable to acretion events on a young star (Ryutov et al. 2000). We verified in particular that our setup is representative of a high plasma $\beta(\geq 1)$ Classical T Tauri Stars (CTTSs) accretion case (Revet et al. 2019). We experimentally demonstrated the formation of a shell of dense (and optically thick when scaled to astrophysical conditions) plasma that envelopes the core's post-shock region and absorbs the Xrays arising from the central core. Such enveloping leads to the decreasing of X-ray flux from the star, which is consistent with discrepancies between astrophysical observations and numerical predictions in the X-ray domain (Bonito et al. 2014).

We studied this astrophysical phenomenon in a scaled laboratory experiment using laser-produced plasma streams impacting onto a tilted obstacle. These streams thus represent accretion material impacting onto a star in a slanted configuration. The formation of such streams takes place with the help of a large-scale, quasi-static $B$-field, perpendicular to the surface of the target irradiated by the laser (Albertazzi et al. 2014; Higginson et al. 2017a). In this work, we explore the oblique incidence of incoming laser-created plasma streams to the secondary obstacle target mimicking the surface of the star.

The paper is organized as follows. In Sect. 2, we describe the setup and approach of the laboratory experiment, and discuss the scalability between the laboratory and the accretion process; in Sect. 3, we present the results of the experiment and the results of the simulations and synthesis of X-ray emission; in Sect. 4, we describe the magnetohydrodynamics (MHD) model of accretion impacts in young stars, the numerical setup, the synthesis of the $\mathrm{X}$-ray emission from the MHD model, and the spectral analysis; and in Sect. 5, we discuss the results and draw our conclusions.

\section{Laboratory experiment approach}

\subsection{Design of the experiment and of the plasma diagnostics}

The experiments were conducted at the ELFIE laser facility (Zou et al. (2008)); the set-up is shown in Fig. 1. An optical laser pulse with energy up to $50 \mathrm{~J}, 0.6 \mathrm{~ns}$ full width at half maximum duration at the wavelength $1057 \mathrm{~nm}$ was focused onto the surface of a Teflon $\left(\mathrm{CF}_{2}\right)$ target. A lens with $2.2 \mathrm{~m}$ focal length and a random phase plate were used, providing a $0.5 \mathrm{~mm}$ focal spot diameter beam with intensity up to $4 \times 10^{13} \mathrm{~W} \mathrm{~cm}^{-2}$. After irradiating the target by the laser, a hot long plasma stream was collimated by applying a large-scale quasi-static homogeneous $B$-field, aligned with the main expansion axis of the plasma, with a strength of up to $30 \mathrm{~T}$, which was oriented normally to the target surface (Higginson et al. 2017b), as shown in Fig. 1.

This plasma stream hit the secondary obstacle PET $\left(\left(\mathrm{C}_{10} \mathrm{H}_{8} \mathrm{O}_{4}\right)_{n}\right)$ target, which was positioned at a distance of 18 $\mathrm{mm}$ from the first target. The incoming plasma stream had a constant diameter of $1.0 \mathrm{~mm}$ at the location of the obstacle, and over more than $100 \mathrm{~ns}$, it impacts the obstacle with a plasma

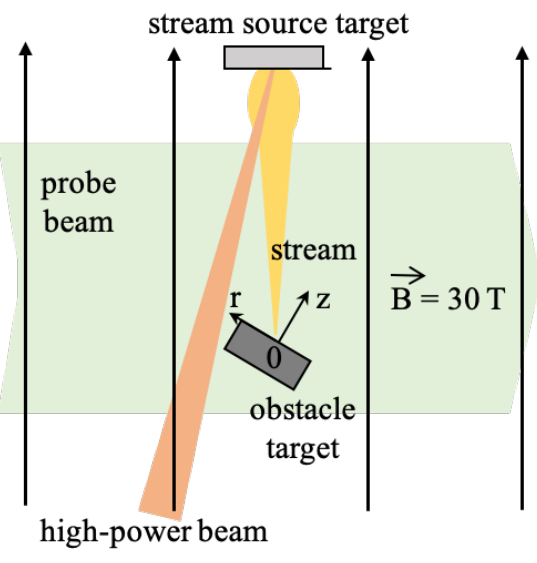

Fig. 1. Schematic view of the experimental set-up.

electron density $\left(n_{\mathrm{e}} \approx 2 \times 10^{18} \mathrm{~cm}^{-3}\right)$; the measured plasma electron temperature of the incoming stream was $10 \mathrm{eV}$ or $0.1 \mathrm{MK}$. The angle at which the incoming plasma stream impacts the secondary target was set, with respect to the obstacle target normal, to $0^{\circ}, 15^{\circ}, 30^{\circ}$, and $45^{\circ}$ for different shots.

To fully investigate the plasma plume originating from the obstacle under the incoming stream impact, we used several complementary diagnostics, allowing us to measure plasma parameters in all principal regions of the structure.

Optical interferometry was used to investigate features of plasma propagation at distances above $0.5 \mathrm{~mm}$ from the obstacle target, where the plasma is transparent for the optical probe beam, providing $2 \mathrm{D}$ side-view distributions of the plasma electron density.

The streaked optical pyrometery (SOP) diagnostic was used to record the visible light $(400-600 \mathrm{~nm})$ emitted, in the optically thin regime, by the plasma close to the obstacle target (i.e., in regions of higher density that cannot be accessed by the interferometry diagnostic), since the latter is obscured close to the target due to refraction of the optical probe beam induced by strong density gradients. It can hence give information on the density structure close to the obstacle surface following the impact.

A variable spacing grating (VSG) spectrometer (Kita et al. 1983) and a focusing spectrograph with spatial resolution (FSSR; Faenov et al. 1994) were utilized to record the emissivity of the plasma in the soft X-ray domain: broadband $(0.4-2 \mathrm{keV})$ and narrow $(0.8-0.95 \mathrm{keV})$ spectrum ranges, respectively. As the $\mathrm{SOP}$, it also resolves the plasma emission along the $Z$ axis, but the signal is integrated along the radial $(r)$ axis, as well as integrated in time. However, the emission is spectrally resolved in the range of sensitivity. It can hence inform us of the temperature of the plasma following impact.

\subsection{Scalability}

To demonstrate the scalability between the laboratory experiment and the accretion process, we detail below and in Table 1 the comparison of the parameters of the incoming streams of plasma before impacting the obstacle target or the surface of the star, correspondingly. A more detailed description of the full dynamical plasma structure, including that of the core and of the post-shock shell, can be found in Revet et al. (2017).

Following the approach presented in Ryutov et al. (2000), for two systems to be scalable to each other, they have to be accurately described by the ideal MHD. It means that the Reynolds number (the ratio of the inertial force to the viscous force) 
$R e=L V / v$, where $\mathrm{L}$ is the characteristic spatial scale, $V$ is the flow velocity, and $v$ is the kinematic viscosity; the Magnetic Reynolds number (the ratio of the convection over ohmic dissipation) $R e_{M}=L V / \eta$, where $\eta$ is the magnetic diffusivity; and the Peclet number (the ratio of heat convection to the heat conduction) $P e=L V / \chi$, where $\chi$ is the thermal diffusivity, should all be higher, or even much higher than 1 . As can be seen from Table 1, for both systems, these condition are satisfied.

Two scaling quantities, the Euler number $\left(E u=V(\rho / p)^{1 / 2}\right)$ and the plasma beta $\left(\beta=8 \pi p / B^{2}\right)$, where $\rho$ is the mass density, $p=k_{\mathrm{B}}\left(n_{i} T_{i}+n_{e} T_{e}\right)$ is the thermal pressure $\left(k_{\mathrm{B}}\right.$ is the Boltzmann constant, $n_{i, e}$ and $T_{i, e}$ are the number densities and the temperatures of the ions and the electrons, correspondingly), and $B$ is the magnetic field, have to be similar in order to have two systems scaled to each other and evolve in the same manner (Ryutov 2018). For the laboratory case, the Euler number is 45.4 and the plasma beta is $8.4 \times 10^{-3}$, and for the astrophysical case these numbers are equal to 87.2 and $2.2 \times 10^{-3}$, respectively. So, it is clearly seen that for both systems these numbers are in agreement. From this, we can deduce that the two systems can indeed be scaled to each other.

The speed of the incoming accretion stream approaches $500 \mathrm{~km} \mathrm{~s}^{-1}$ near the surface of the star. Typical velocities of the laboratory plasma vary from 100 to $1000 \mathrm{~km} \mathrm{~s}^{-1}$, and the value of $750 \mathrm{~km} \mathrm{~s}^{-1}$, presented in Table 1 , corresponds to the peak velocity of the highest electron density part of the stream. The characteristic spatial scale of the accretion stream is to the order of tens of thousands kilometers and the typical laboratory scale of the stream is of the order of one millimeter. The density $\rho_{\text {ast }}$ of the accretion stream is $1.1 \times 10^{-11} \mathrm{~g} \mathrm{~cm}^{-3}$ and the laboratory stream density $\rho_{\text {lab }}$ is $1.1 \times 10^{-5} \mathrm{~g} \mathrm{~cm}^{-3}$. From all this, and following Ryutov's analysis, the following scaling factors can be extracted: $a=r_{\text {ast }} / r_{\text {rlab }}=5 \times 10^{4}[\mathrm{~km}] / 0.7[\mathrm{~mm}]=$ $7 \times 10^{10}$, which is the spatial scaling parameter. Similarly, the density scaling parameter is $b=\rho_{\text {ast }} / \rho_{\text {lab }}=1.1 \times 10^{-11}$ $\left[\mathrm{g} \mathrm{cm}^{-3}\right] / 1.1 \times 10^{-5}\left[\mathrm{~g} \mathrm{~cm}^{-3}\right]=10^{-6}$, and the velocity scaling parameter is $c=V_{\text {ast }} / V_{\text {lab }}=500\left[\mathrm{~km} \mathrm{~s}^{-1}\right] / 750\left[\mathrm{~km} \mathrm{~s}^{-1}\right]=0.67$. Therefore, the temporal scaling is $t_{\mathrm{ast}}=(a / c) t_{\mathrm{lab}}$, which means that $100 \mathrm{~ns}$ in the laboratory is equivalent to around $10^{13} \mathrm{~ns}$ or hours in the astrophysical case. The magnetic field scaling gives $B_{\text {ast }}=B_{\text {lab }} c \sqrt{b}$, meaning that $30 \mathrm{~T}$ in the laboratory case corresponds to $2 \times 10^{-2} \mathrm{~T}(200 \mathrm{G})$ in the CTTS case, which is in agreement with the magnetic field strength expected in CTTSs. We also note that a similar correspondence to the laboratory experiments, meaning similar values of the Euler number and of the plasma beta, can be obtained for other sets of CTTS magnetic field and accretion stream density. For example, for a density of $5 \times 10^{10} \mathrm{~cm}^{-3}$, which is in the range of values inferred from the analysis of X-ray observations for accretion impacts producing detectable X-ray emission (e.g. Curran et al. 2011), the plasma $\beta$ investigated in our experiments can be obtained with a magnetic field strength to the order of $10 \mathrm{G}$, similarly to that explored in MHD simulations of accretion impacts in CTTSs with moderate to high plasma $\beta$ values (e.g., Orlando et al. 2010). We underline that the range of CTTS estimated parameters is large, and that, even on a given star, it is expected that a wide range of local magnetic field values and varied accretion stream densities exist.

We stress that there is no scalability between the X-ray emissivity measured in the laboratory case and that of the astrophysical object, as the absorption properties of the two plasmas are dissimilar. The X-ray emissivity measurements performed in the laboratory and which are detailed below are merely used in order to derive the temperature of the laboratory plasma, and to
Table 1. Comparison and scalability between the laboratory plasma stream impacting the obstacle target and the CTTS accretion stream impacting the surface of the star.

\begin{tabular}{|c|c|c|}
\hline & Laboratory & CTTS \\
\hline Material & $\mathrm{CF}_{2}$ (Teflon) & $H$ \\
\hline Charge state $Z$ & 3.94 & 1 \\
\hline Mass number $A$ & 17.32 & 1.28 \\
\hline$B$-field [T] & 30 & $2 \times 10^{-2}$ \\
\hline Spatial scale $[\mathrm{cm}]$ & 0.07 & $5 \times 10^{9}$ \\
\hline Electron density $\left[\mathrm{cm}^{-3}\right]$ & $1.5 \times 10^{18}$ & $5 \times 10^{12}$ \\
\hline Ion density $\left[\mathrm{cm}^{-3}\right]$ & $3.8 \times 10^{17}$ & $5 \times 10^{12}$ \\
\hline Density $\left[\mathrm{g} \mathrm{cm}^{-3}\right]$ & $1.1 \times 10^{-5}$ & $1.1 \times 10^{-11}$ \\
\hline $\mathrm{Te}[\mathrm{eV}]$ & 10 & 0.22 \\
\hline $\mathrm{Ti}[\mathrm{eV}]$ & 10 & 0.22 \\
\hline Flow velocity $\left[\mathrm{km} \mathrm{s}^{-1}\right]$ & 750 & 500 \\
\hline Sound velocity $\left[\mathrm{km} \mathrm{s}^{-1}\right]$ & 21.3 & 7.4 \\
\hline Alfven velocity $\left[\mathrm{km} \mathrm{s}^{-1}\right]$ & 254.8 & 172.4 \\
\hline Electron mean free path $[\mathrm{cm}]$ & $4.1 \times 10^{-5}$ & 0.02 \\
\hline Electron collision time [ns] & $3 \times 10^{-4}$ & 1.1 \\
\hline Ion mean free path $[\mathrm{cm}]$ & $3.7 \times 10^{-6}$ & 0.03 \\
\hline Ion collision time [ns] & $5 \times 10^{-3}$ & 74.5 \\
\hline Magnetic diffusion time [ns] & $0.8 \times 10^{3}$ & $4.8 \times 10^{22}$ \\
\hline Electron Larmor radius $[\mathrm{cm}]$ & $2.5 \times 10^{-6}$ & $5.6 \times 10^{-4}$ \\
\hline Electron gyrofrequency $\left[\mathrm{s}^{-1}\right]$ & $8.4 \times 10^{12}$ & $5.6 \times 10^{9}$ \\
\hline Ion Larmor radius $[\mathrm{cm}]$ & $1.1 \times 10^{-4}$ & 0.027 \\
\hline Ion gyrofrequency $\left[\mathrm{s}^{-1}\right]$ & $1 \times 10^{9}$ & $2.4 \times 10^{6}$ \\
\hline Electron magnetization & 16.3 & 38.2 \\
\hline Ion magnetization & 0.03 & 1.1 \\
\hline Mach number & 35.2 & 67.5 \\
\hline Alfven Mach number & 2.9 & 2.9 \\
\hline Reynolds number & $3.1 \times 10^{3}$ & $1.1 \times 10^{12}$ \\
\hline Magnetic Reynolds number & $0.86 \times 10^{3}$ & $4.8 \times 10^{11}$ \\
\hline Peclet number & 17 & $2.3 \times 10^{10}$ \\
\hline Euler number & 45.4 & 87.2 \\
\hline$\beta$ & $8.4 \times 10^{-3}$ & $2.2 \times 10^{-3}$ \\
\hline
\end{tabular}

confirm the lack of confinement of accreted plasma induced by slanting the accretion column.

\section{Experimental results}

\subsection{Optical interferometry}

The experiment reveals, for slanted impact, a strong asymmetry of the plasma structure following impact, with strong lateral leakage of matter away from the impact point.

Such evolution of the plasma during the impact of the plasma flow on the obstacle was measured by means of a MachZehnder-based interferometer from the time $(t=0 \mathrm{~ns})$ when the plasma stream reaches the secondary target, and up to $t=$ $118 \mathrm{~ns}$, which is our limit of observation corresponding to $1680 \mathrm{~s}$ (half an hour) in the astrophysical case. This number was calculated using the laboratory-astrophysical scaling detailed in (Revet et al. 2017), which used the same laboratory platform as the present experiment. The probe beam used for the interferometer was a compressed beam with $100 \mathrm{~mJ}$ energy and 350 fs duration at the fundamental wavelength $1057 \mathrm{~nm}$, combined collinearly with its second-harmonic pulse having $30 \mathrm{~mJ}$ energy and the same duration but at a wavelength of $528.5 \mathrm{~nm}$. A Glan prism was used to polarizationaly split both beams into two 

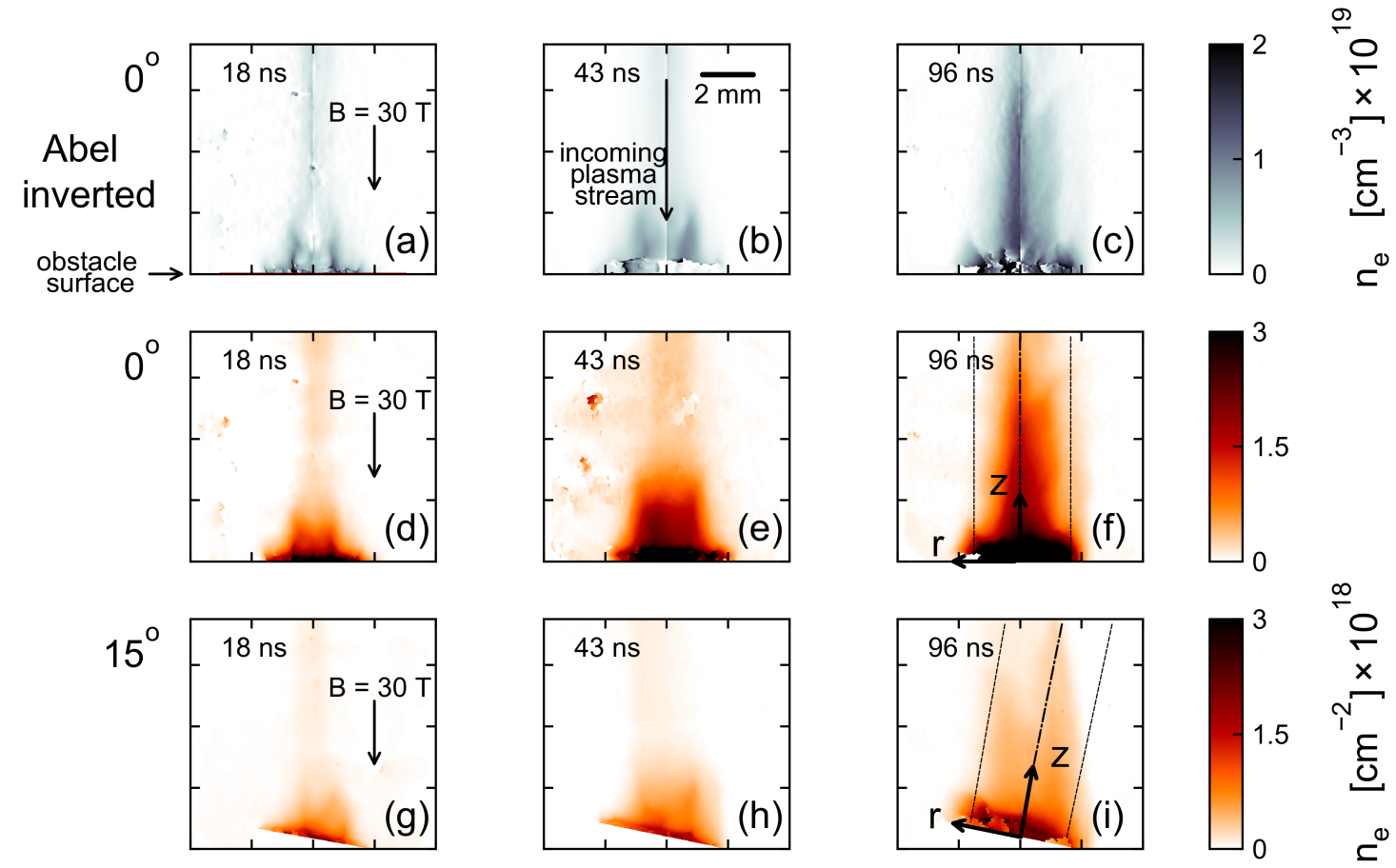

$\stackrel{\infty}{\sim}$
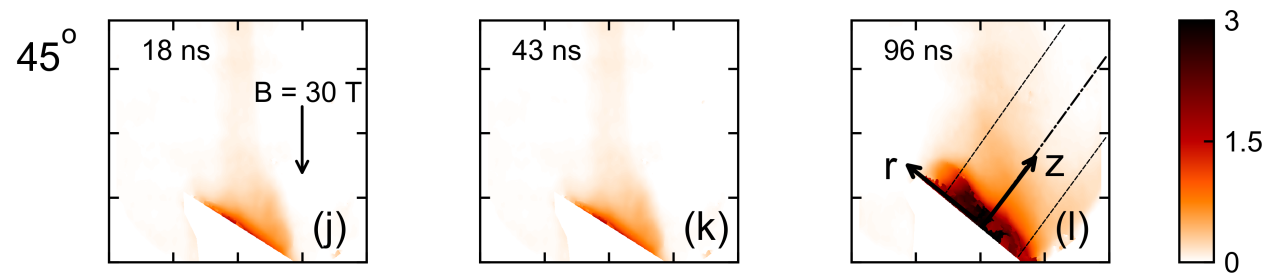

Fig. 2. Evolution of plasma electron density in time (18 ns, $43 \mathrm{~ns}$, and $96 \mathrm{~ns})$ : $(a)-(c)$ : Abel inverted volumetric density profiles for the normal incoming stream impact; $(d)-(f)$ : $2 \mathrm{D}$, line-integrated, side-view distributions of the plasma electron density for normal incoming stream, corresponding to the same images as shown in panels a-c; $(g)-(i)$ : same for $15^{\circ}$ oblique stream incidence, $(j)-(l)$ : same for $45^{\circ}$ oblique stream incidence.

replicas ( $\mathrm{S}$ and $\mathrm{P}$ ), delay the $\mathrm{S}$ with respect to the $\mathrm{P}$, and then recombine them collinearly (see Higginson et al. 2017b). Thus, before probing the plasma, we had four temporally separated beams delayed relative to each other by $12 \mathrm{~ns}$, which provided four interferograms in one shot.

Plasma density snapshots for three consecutive points in time (18 ns, $43 \mathrm{~ns}$, and $96 \mathrm{~ns}$ ) following the interaction of the incoming stream with the obstacle target are presented in Fig. 2.

The initial moment $(t=0)$ and zero point $(x=0)$ matches to the time and place of the stream tip arrival to the surface of the obstacle. Figures $2 \mathrm{a}-\mathrm{c}$ show the volumetric plasma electron density profiles retrieved by Abel inversion from the experimental phase maps obtained using time-resolved optical interferometry for the normal impact case. The corresponding 2D distributions of the line-of-sight integrated density of the plasma are presented in Figs. 2d-f. Two-dimensional distributions for oblique incidence angles $15^{\circ}$ and $45^{\circ}$ are shown in Figs. 2g-i and Figs. 2j-1, correspondingly.

For the normal impact case, consistently with previous results (Revet et al. 2017), we see the progressive formation of a symmetrical plasma structure, meaning a characteristic shocked core within the stream and surrounded by a dense envelope, surrounding the incoming stream. This structure was presented in detail in our previous work (Revet et al. 2017). And for the slanted impact case, we clearly observe the development of an asymmetric plasma density distribution with a denser plasma structure on the side of the reduced angle of impact. As can also be seen in Fig. 2, the post-shocked plasma flow has a tendency to follow the magnetic field lines. Thus, larger obstacle tilt angles demonstrate less confined and more asymmetric plasma distributions.

The lineouts of the 2D electron plasma density along the axis perpendicular to the surface of the obstacle target for $0^{\circ}, 15^{\circ}$, and $45^{\circ}$ stream impact at $96 \mathrm{~ns}$ are presented in Fig. 3. We chose to make lineouts perpendicularly to the target surface to characterize how the rotation affects the development of the shoulders of the envelope close to the target, around the column. The positive lineout crosses the incoming stream, but it is not dense compared to the ejecta in this region. They demonstrate characteristic features of propagating plasma (see dashed and dot-dashed lines in Figs. 2f, i, l). The zero points of the axes $(z=0, r=0)$ correspond to the stream tip arrival on the surface of the obstacle. The $Z$-axis corresponds to the axis of symmetry for normal stream incidence case (Fig. 2f). Off-axis slices along $Z$ correspond to the positions of the symmetrical edges of the "wings" of the postshock front for the normal impact (Fig. 2f). For slanted impacts $\left(15^{\circ}\right.$ and $\left.45^{\circ}\right)$ such wings evolve strongly asymmetrically and become less distinguishable with time.

For the normal impact case, the density distribution along the zero axis is much higher compared to the density profiles along the wings of the post-shocked plasma, which are monotonously decreasing and both have quite similar values. 

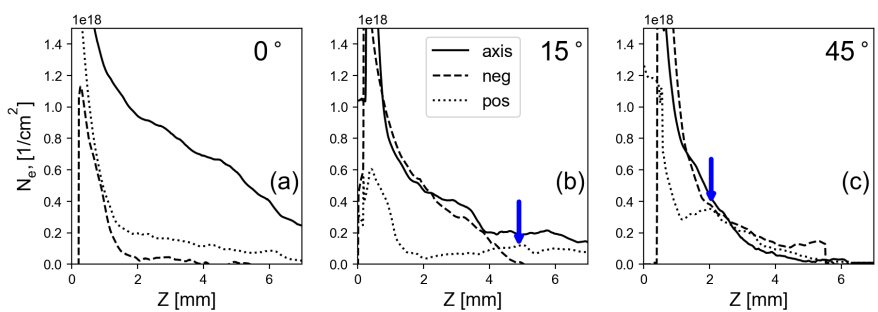

Fig. 3. Profiles of the line-integrated electron plasma density at $96 \mathrm{~ns}$ along the $Z$-axis (solid line), along $r=-0.92 \mathrm{~mm}$ post-shock wing (dashed line) and along $r=1.17 \mathrm{~mm}$ post-shock wing (dotted line). The various lines are directly represented in the images shown in Figs. 2f,i,l.

For the tilted cases, the positive off-axis density profile (dotted lines) exhibit a noticeable "hump" in the profile (see the arrows pointing to it in Fig. 3), which appears closer to the obstacle target with increasing tilt (about $5.0 \mathrm{~mm}$ from the target for $15^{\circ}$ (Fig. 3b) and $2.0 \mathrm{~mm}$ for $45^{\circ}$ (Fig. 3c).

The negative off-axis density profiles for all cases decrease monotonously. For the tilted cases, the positive and the negative off-axis density profiles notably differ from each other compared to the symmetrical normal impact case. The on-axis profile in Fig. 3 decreases strongly for the slanted case due to the weaker shock structure and lack of plasma column confinement, while the negative off-axis lineout strongly increases, which directly shows evidence of plasma leakage in the negative direction.

The zero axis density profiles for normal and tilted cases also demonstrate a "plateau and sharp decrease" structure of the density front at a distance from the obstacle surface. Such a structure becomes closer to the obstacle surface with increasing tilt angle (about $5.5 \mathrm{~mm}$ for $0^{\circ}$ (Fig. 3a), $4 \mathrm{~mm}$ for $15^{\circ}$ (Fig. $3 \mathrm{~b}$ ), $1.5 \mathrm{~mm}$ for $45^{\circ}$ (Fig. $\left.3 \mathrm{c}\right)$ ). This quantitatively shows the increasing difficulty in refocusing matter on the axis, following the impact of increasingly slanted accretion columns. For the tilted cases, the difference between the values of the on-axis density profile and that of the wings becomes clearly less pronounced and decreases faster with increasing tilt angle. This is a direct representation of the much weaker shock and less confinement in the slanted case.

Reducing the magnetic field strength to $20 \mathrm{~T}$, as shown in Fig. 4, we observe similar effects, except with wider overall structures.

\subsection{Visible self-emission of plasma}

In practice, the interferometry diagnostic is limited to probing density regions up to $2-5 \times 10^{19} \mathrm{~cm}^{-3}$. Due to refraction of the probe beam at distances closer than $1 \mathrm{~mm}$ to the target, surface interferometry cannot provide correct values of electron plasma density in this region. Self-emitted light is however able to escape from such dense plasma since the plasma is still optically thin. It is thus there that the SOP diagnostic is useful to give information about dense plasma regions close to the obstacle surface. The SOP diagnostic records, in the visible band (400$600 \mathrm{~nm}$ ) and resolved in time, the emission of a thin ( $2 \mathrm{~mm}$ wide) slice of plasma along the $Z$-axis, centered at $r=0$.

As shown in Fig. 5, the SOP diagnostic can record emission of the plasma as close as $80 \mu \mathrm{m}$ from the obstacle surface (i.e., in regions corresponding to densities going up to $10^{21} \mathrm{~cm}^{-3}$ ) according to 3D simulations of the experiment (Revet et al. 2017). The SOP emission shown in Fig. 5 for various inclination of the obstacle target clearly shows that the density in the shocked region close to the obstacle surface is strongly reduced as the inclination of the obstacle increases. Indeed, in this regime of optically thin plasma, we expect that the total emissivity of the plasma is proportional to the density of the emitting plasma. Here, we present the SOP data for $20 \mathrm{~T}$, because of the lack of $30 \mathrm{~T}$ SOP data, but since interferometry for both $20 \mathrm{~T}$ and $30 \mathrm{~T}$ cases represents almost the same features, comparative decreasing of plasma emission with increasing of the obstacle target tilt should be the same in both cases.

Therefore, with the help of the interferometry and the SOP, we have complementary diagnostics for low-density and highdensity electron plasma, representing lateral ejection of material, and subsequently, less axial confinement of plasma under oblique impact.

\subsection{X-ray self-emission of plasma}

\subsubsection{Experimental results}

Similarly to the SOP, the VSG diagnostic records the laboratory plasma emissivity, but this time in a broadband $\mathrm{X}$-ray range $(0.4-2 \mathrm{keV})$. It also resolves the plasma emission along the $Z$ axis, but the signal is integrated along the radial $(r)$ axis, as well as being integrated in time. However, the emission is spectrally resolved in the range of sensitivity. We used the same setup as described in Kita et al. (1983) with the concave grating with an incidence angle of $87^{\circ}$ and nominal groove number of 1200 grooves $\mathrm{mm}^{-1}$. The detector used was a FuijiFilm TR-type image plate. In front of the image plate was a sheet of aluminized plastic to serve as a light-tight filter that also created absorption edges that were used to calibrate the energy dispersion. For the deconvolution of the VSG data, the dependencies of the image plate sensitivity (Haugh et al. 2013) and of the reflectivity of the grating (Park et al. 2010) on the energy of photons were both taken into an account.

The VSG emission shown in Fig. 6 for various inclinations of the obstacle target clearly shows, complementary to the SOP diagnostics, that: (1) the confinement of the plasma along the $Z$ axis strongly decreases as the incoming stream is increasingly slanted. This is evidenced by the strong decrease in the amplitude of the emission along $Z$ as the obstacle inclination increases. Secondly, (2) the plasma near the obstacle gets colder as the incoming stream is increasingly slanted, as evidenced by the progressive drift of the signal toward lower photon energies. This is consistent with a lesser capability of the plasma to be confined due to the increased angle between the magnetic field and the incoming stream axis.

We note that the VSG is equipped with a vertical slit (perpendicular to the incoming stream axis, $Z$, which, in the laboratory, is horizontal) in order to allow spatial resolution along the $Z$ axis. The grating has its lines in the horizontal direction. Hence, we have horizontal spatial resolution and vertical spectral resolution on the VSG detector. However, since we used a slit instead of a pinhole at the VSG entrance, the spectrum in the vertical axis is not purely spectral and is mixed with space (various emitting points in the plasma at different heights (along the axis r) will appear at various heights on the IP). Another effect of using a slit is that the tilted targets also appear tilted in the IP detector, as is apparent in Fig. 6. The mixing of space and spectrum induces some smearing on the recorded spectra, in such a way that we do not have enough spectral resolution to resolve exact transition lines. However, the observed spectral changes, when tilting the obstacle, have to be compared to the size of the smearing induced by the source height. In real space, the spatial width of the plasma source in the vertical direction is $1-2 \mathrm{~mm}$ 

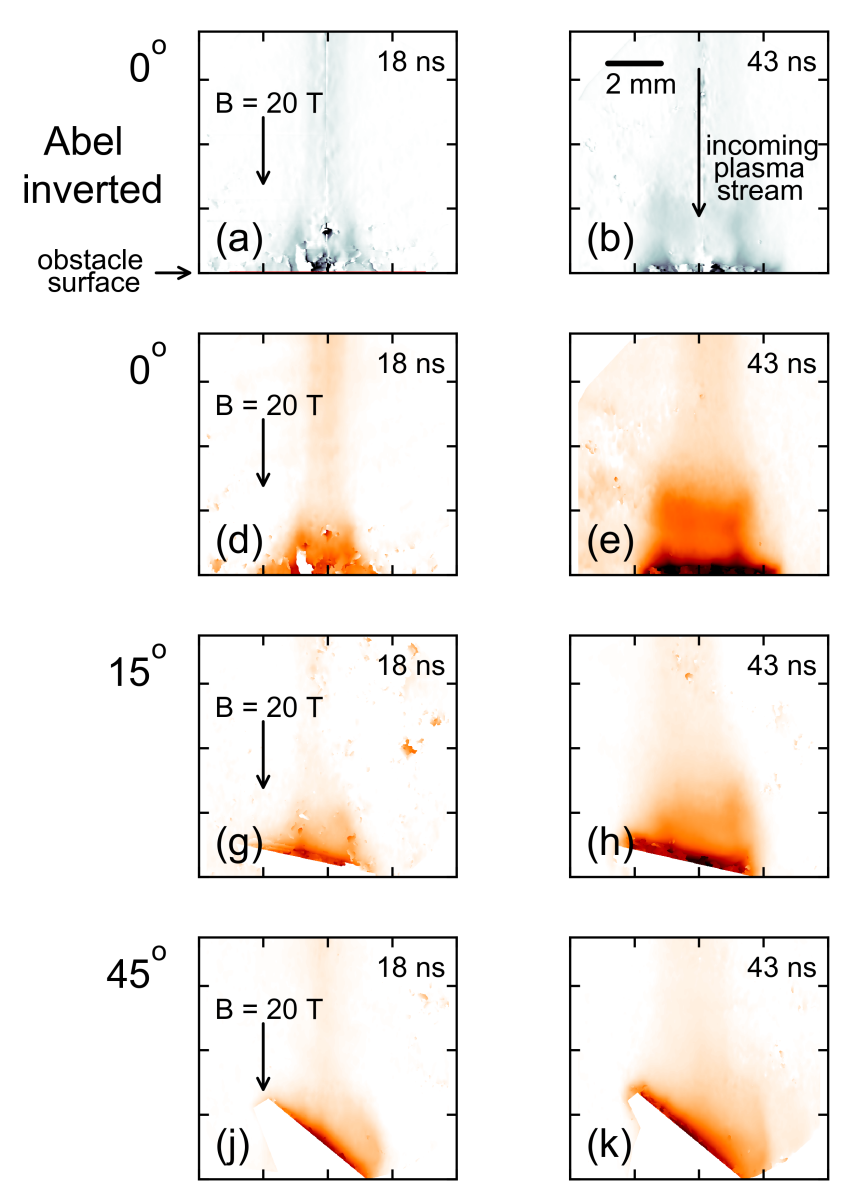

$\stackrel{9}{\leftarrow}$
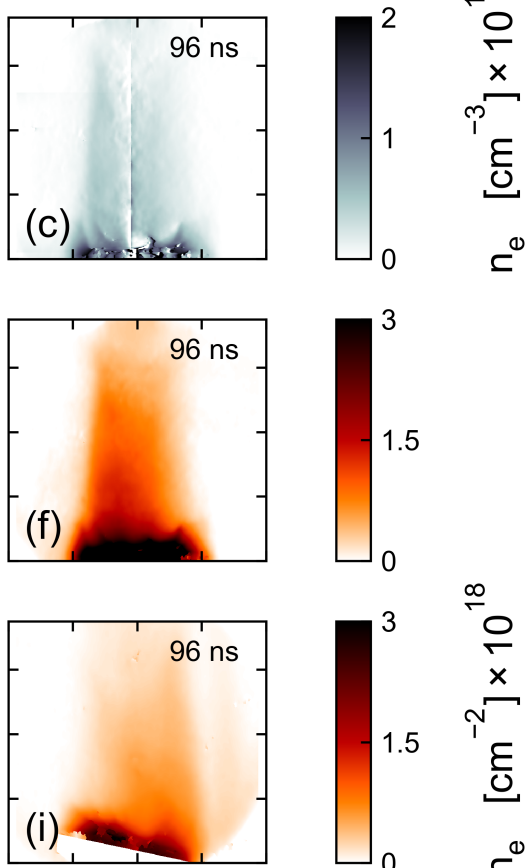

$\stackrel{\infty}{\sim}$
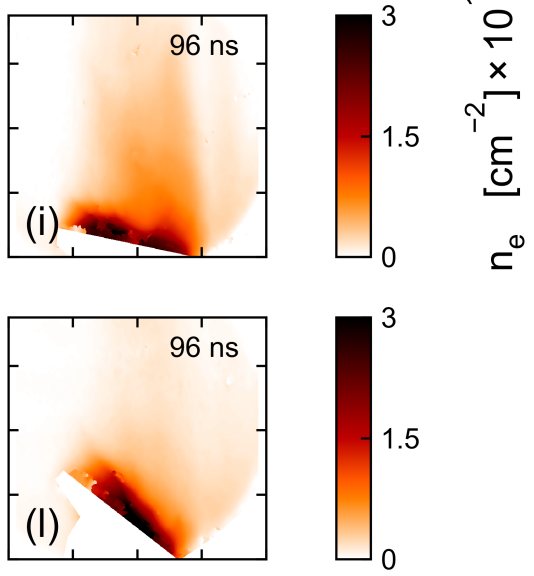

Fig. 4. Evolution of plasma electron density with the same arrangement as for Fig. 2 but for lower $B=20 \mathrm{~T}$.
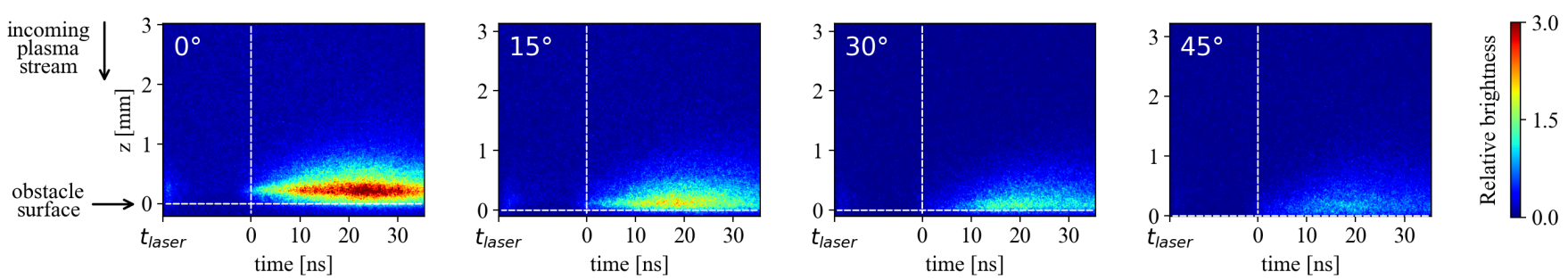

Fig. 5. Visible self-emission of plasma measured by the SOP diagnostic for $B=20 \mathrm{~T}$. The color bar, which represents the emissivity in arbitrary units, is the same for all images.

large, which transposes in the same distance on the detector as there is a magnification of one through the VSG. This has to be compared to the typical extent of the spectral features shown in Fig. 6, which are to the order of $6-8 \mathrm{~mm}$ on the detector (i.e., quite large in comparison to the smearing induced by the source size).

The FSSR, which has a much better spectral resolution, but over a smaller bandwidth, allows us to resolve individual lines. A spectrum recorded by the FSSR is shown in Fig. 7 for the emission close to the obstacle target surface (around $0.25 \mathrm{~mm}$ ) for the 0 degree (normal incidence) case. We can use such spectrum to measure the electron temperature and density in a similar way as described in Revet et al. (2017), Filippov et al. (2019). The plasma parameters of the hot cocoon surrounding the colder core of the impact of the incoming stream, as well as that of the core are, respectively, deduced to be $350 \mathrm{eV} / 3 \times 10^{18} \mathrm{~cm}^{-3}$ and $50 \mathrm{eV} / 3 \times 10^{19} \mathrm{~cm}^{-3}$. Close to the obstacle, we also note the observation of oxygen lines in the spectrum, having a high quantum number $\mathrm{n}$, thus confirming that the plasma temperature is high. However, the relative abundance of $\mathrm{O}$ in the plasma, due to the mixing of the incoming stream plasma and of that of the obstacle, is unknown. To further the analysis of the plasma parameters for different obstacle orientations, we turned to using the VSG spectrometer, despite its low spectral sensitivity, taking advantage of its capability to record emissivity over a larger spectral range. This is what is discussed in the next section.

\subsubsection{Numerical modeling of the laboratory plasma X-ray emissivity}

We modeled the plasma emissivity over a broad spectral range in order to be able to infer the plasma temperature from the VSG 

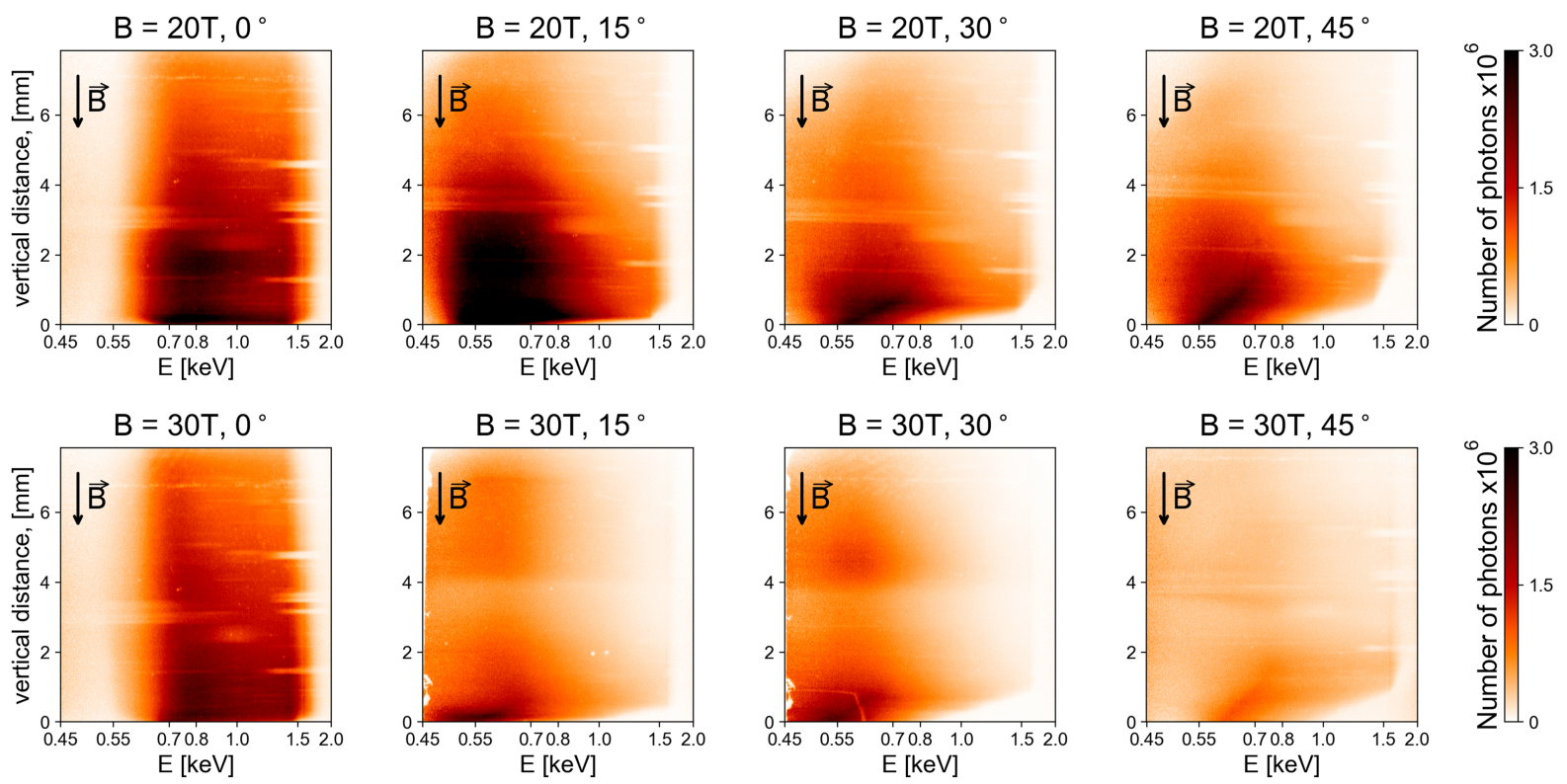

Fig. 6. Energy spectra of plasma self-emission recorded in the soft X-ray, as measured by the VSG diagnostic for $B=20 \mathrm{~T}$ (top row) and $B=30 \mathrm{~T}$ (bottom row; in the $30 \mathrm{~T}$ case for $15^{\circ}$ and $30^{\circ}$ the lighter band around $z=3-4 \mathrm{~mm}$ is an artefact due to a local loss of sensitivity of the detector which was used for these shots). These are raw data corrected for the detector (image plate) response to the X-rays and for the grating reflectivity, which is dependant on the X-ray energy (see text).

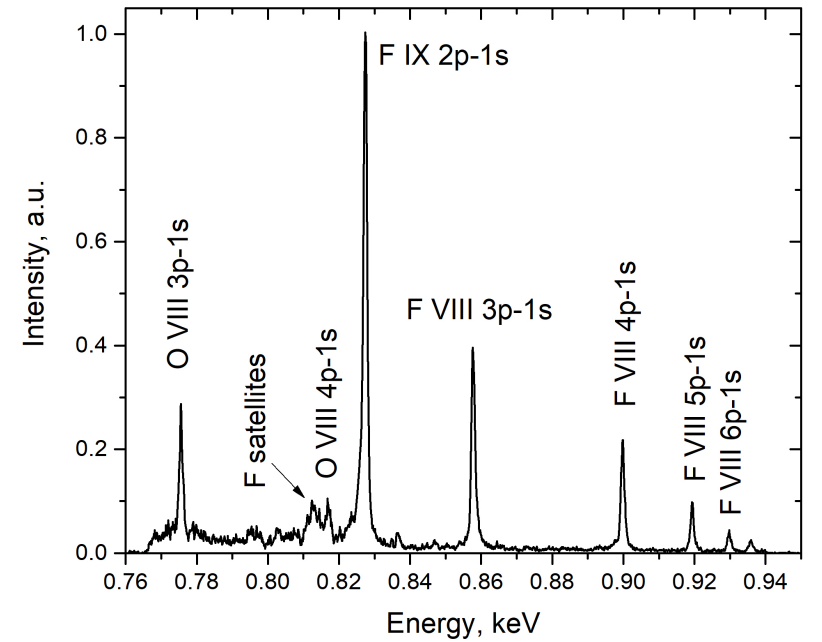

Fig. 7. Typical spectrum measured by FSSR spectrometer for the case of normal incidence. The FSSR line of sight was perpendicular to the plane of Fig. 1 with a spatial resolution along the plasma expansion axis (Z-axis). The observation of the $\mathrm{H}$-like oxygen transition confirms that the electron temperature close to the target is high.

spectra. Since the recorded VSG spectra are integrated along the radial axis as well as in time, many different plasma conditions will have therefore contributed to the emissivity. However, even with insufficient spectral resolution of the VSG spectrometer, the comparison with numerical synthetic spectra, as discussed below, may provide information about the maximum temperatures reached in the plasma plume. With that purpose, we carried out numerical simulations of synthetic spectra of $\mathrm{CF}_{2}$ plasmas using the MIXKIP/RAPCAL codes (Rodriguez et al. 2008; Espinosa et al. 2017). For the calculations, an electron density of $10^{19} \mathrm{~cm}^{-3}$ and a plasma length of $1.5 \mathrm{~mm}$ were used. According to Fig. 2, both average values are reasonable to model the plasma close to the target surface $(z=1.5 \mathrm{~mm})$. Moreover, since there is no time resolution, the synthetic spectrum for a given electron temperature was obtained by adding those calculated for temperatures between $5 \mathrm{eV}$ and the given temperature. To illustrate the results, the situation in which the strength of the magnetic field is $30 \mathrm{~T}$ and $z=1.5 \mathrm{~mm}$ was selected.

Furthermore, to compare with the experiment, we focused on the situation of the normal impact case and of the $45^{\circ}$ oblique stream incidence. In Fig. 8, we see the experimental lineouts corresponding to the VSG spectra recorded in these two cases, i.e. these are the lineouts at the position $z=1.5 \mathrm{~mm}$ of the raw data shown in Fig. 6, bottom row: the left-most (normal incidence) and right-most ( $45^{\circ}$ oblique stream incidence) images. We note that we discuss here, in light of the numerical modeling detailed below, only the features of these experimental spectra below $1 \mathrm{keV}$ photon energy, since it is likely that for higher photon energies, the emission could originate from a recombination continuum.

The emission lines that can be modeled for various plasma temperatures are presented in Fig. 9. Three temperature ranges are modelled: 210-240 eV (Fig. 9a), 380-390 eV (Fig. 9b), and 400-410 eV (Fig. 9c). One can observe that between these three cases, the ratio of the various groups of lines varies.

For the lowest considered temperatures, 210-240 eV (Fig. 9(a)), one can see (1) that the relative weight of the spectral intensities corresponding to the $2 p-1$ s transitions of $F$ IX ion is quite lower than that of the $1 \mathrm{~s} 2 \mathrm{p}-1 \mathrm{~s}^{2}$ transitions of F VIII ion. This is contrary to what happens at higher temperatures. Still, for the lowest considered temperatures, one observes (2) that the contributions provided by the transitions of the $\mathrm{C}$ VI ion below $0.5 \mathrm{keV}$ photon energy range are of similar intensities to those of the $2 \mathrm{p}-1 \mathrm{~s}$ transitions of F IX ion (around $0.83 \mathrm{keV}$ photon energy). These two observations best match the variation of the spectral intensity observed for the $45^{\circ}$ case. This suggests that the plasma temperature is low in this case. However, in the normal incidence case, the situation seems different and would suggest a higher temperature. Indeed, in that case, Fig. 8 shows (1) that the spectral intensity around $0.5 \mathrm{keV}$ is much smaller in comparison to that around $0.75 \mathrm{keV}$. Moreover, contrary to the $45^{\circ}$ case, (2) the spectral intensities from 0.75 to $0.85 \mathrm{keV}$ are 


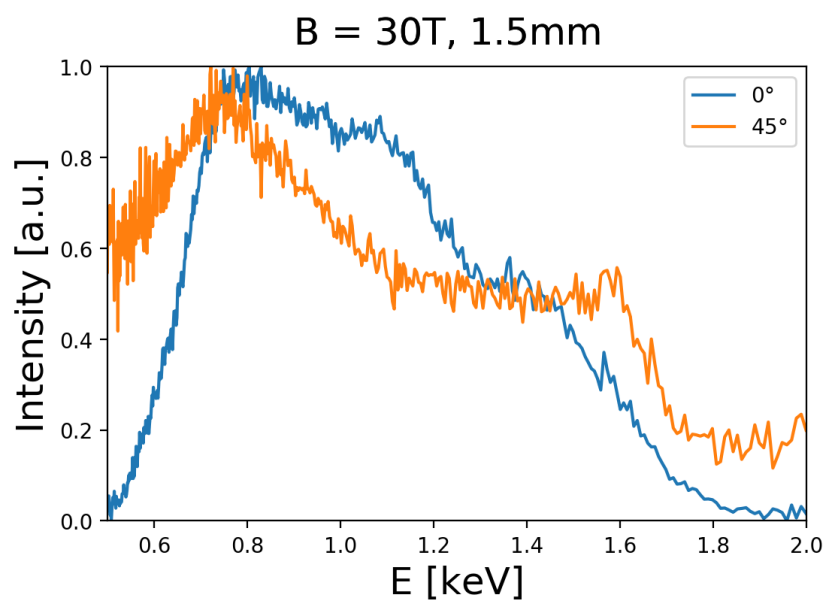

Fig. 8. Spectral lineouts of experimental X-ray emissivity spectra shown in Fig. 6. The lineouts correspond to the images shown in the bottom row of Fig. 6 (i.e., in the case of $B=30 \mathrm{~T}$, for $0^{\circ}$ and $45^{\circ}$ angles). The lineouts are taken at the position $z=1.5 \mathrm{~mm}$.

quite similar. All this is best matched by the case of the hottest considered plasma in the simulations, meaning the one in Fig. 9c. Indeed, in that case, the intensities around $0.8 \mathrm{keV}$, corresponding to the $2 p-1$ s transitions of F IX ion and the $1 s 2 p-1 s^{2}$ transitions of F VIII ion, have similar amplitudes, and the contribution of the transitions of the C VI ion is low. Therefore, this suggests that electron temperatures around $390-410 \mathrm{eV}$ are reached for the overall plasma plume in the normal incidence case.

\section{Modeling accretion impacts on young stars}

\subsection{The MHD model}

The results of the experiment were compared with MHD simulations describing the final propagation of an accretion stream through the atmosphere of a CTTS and its impact on the chromosphere of the young stellar object. To this end, we adopted the model of Orlando et al. (2010), which assumes a stream of plasma downflowing along magnetic field lines that link the circumstellar disk to the stellar surface. The magnetic field is assumed to be uniform, but, at variance with the model of Orlando et al. (2010), the field can have an angle smaller than $90^{\circ}$ with respect to the stellar surface. The computational domain includes only the portion of the stellar atmosphere where the impact occurs. The model assumes a fully ionized plasma with a ratio of specific heats $\gamma=5 / 3$, and accounts for the effects of gravity, radiative cooling, and thermal conduction (including the effects of heat flux saturation). The stream impact is modeled by numerically solving the time-dependent MHD equations of mass, momentum, and energy conservation in nondimensional conservative form:

$\frac{\partial \rho}{\partial t}+\nabla \cdot(\rho \boldsymbol{u})=0$

$\frac{\partial \rho \boldsymbol{u}}{\partial t}+\nabla \cdot(\rho \boldsymbol{u} \boldsymbol{u}-\boldsymbol{B} \boldsymbol{B})+\nabla P_{*}=\rho \boldsymbol{g}$,

$\frac{\partial \rho E}{\partial t}+\nabla \cdot\left[\boldsymbol{u}\left(\rho E+P_{*}\right)-\boldsymbol{B}(\boldsymbol{u} \cdot \boldsymbol{B})\right]=\rho \boldsymbol{u} \cdot \boldsymbol{g}-\nabla \cdot \boldsymbol{F}_{\mathrm{c}}-n_{\mathrm{e}} n_{\mathrm{H}} \Lambda(T)$,

$\frac{\partial \boldsymbol{B}}{\partial t}+\nabla \cdot(\boldsymbol{u B}-\boldsymbol{B} \boldsymbol{u})=0$
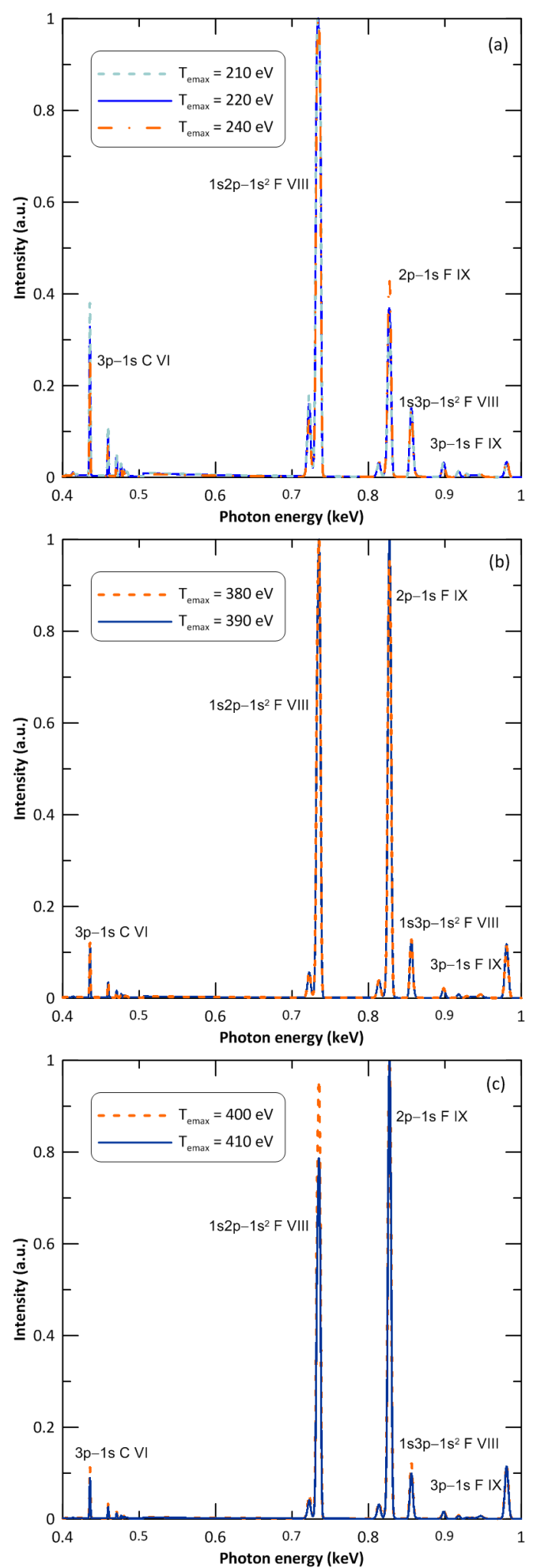

Fig. 9. Synthetic spectra of $\mathrm{CF}_{2}$ plasma obtained for different electron temperatures and for an electron density of $10^{19} \mathrm{~cm}^{-3}$ and a plasma length of $1.5 \mathrm{~mm}$.

where

$P_{*}=P+\frac{B^{2}}{2} \quad$ and $\quad E=\epsilon+\frac{1}{2} u^{2}+\frac{1}{2} \frac{B^{2}}{\rho}$

are the total pressure and the total gas energy (internal energy, $\epsilon$, kinetic energy, and magnetic energy), respectively; $t$ is the time; 
K. Burdonov et al.: Laboratory evidence for asymmetric accretion structure upon slanted matter impact in young stars

$\rho=\mu m_{\mathrm{H}} n_{\mathrm{H}}$ is the mass density; $\mu=1.28$ is the mean atomic mass (assuming metal abundances of 0.5 of the solar values: Anders \& Grevesse 1989); $m_{H}$ is the mass of the hydrogen atom; $n_{\mathrm{H}}$ is the hydrogen number density; $\boldsymbol{u}$ is the gas velocity, $g$ is the gravity; $T$ is the temperature; $\boldsymbol{B}$ is the magnetic field; $\boldsymbol{F}_{\mathrm{c}}$ is the conductive flux, and $\Lambda(T)$ represents the optically thin radiative losses per unit emission measure derived with the PINTofALE spectral code (Kashyap \& Drake 2000) with the APED atomic line database (Smith et al. 2001), assuming the same metal abundances as in Orlando et al. (2010) (as deduced from X-ray observations of CTTSs; Telleschi et al. 2007). We use the ideal gas law, $P=(\gamma-1) \rho \epsilon$.

The thermal conductivity is highly anisotropic due to the presence of the stellar magnetic field: it is strongly reduced in the direction transverse to the field (e.g., Spitzer 1962). Furthermore, the model includes the classical and the saturated conduction regime. Thus, we treated the thermal flux as in Orlando et al. (2008), by splitting the thermal flux into two components, along and across the magnetic field lines, $\boldsymbol{F}_{\mathrm{c}}=F_{\|} \boldsymbol{i}+F_{\perp} \boldsymbol{j}$, where

$$
\begin{aligned}
& F_{\|}=\left(\frac{1}{\left[q_{\mathrm{spi}}\right]_{\|}}+\frac{1}{\left[q_{\mathrm{sat}}\right]_{\|}}\right)^{-1}, \\
& F_{\perp}=\left(\frac{1}{\left[q_{\mathrm{spi}}\right]_{\perp}}+\frac{1}{\left[q_{\mathrm{sat}}\right]_{\perp}}\right)^{-1} .
\end{aligned}
$$

$\left[q_{\text {spi }}\right]_{\|}$and $\left[q_{\text {spi }}\right]_{\perp}$ are the classical conductive flux along and across the magnetic field lines following (Spitzer 1962), and $\left[q_{\text {sat }}\right]_{\|}$and $\left[q_{\text {sat }}\right]_{\perp}$ are the saturated flux along and across the magnetic field lines following (Cowie \& McKee 1977). Equations (5) allow for a smooth transition between the classical and saturated conduction regime (see Dalton \& Balbus 1993; Orlando et al. 2008).

The gravity is calculated assuming the star mass $M=1.2 M_{\odot}$ and the star radius $R=1.3 R_{\odot}$, which are appropriate for the CTTS MP Mus (see Argiroffi et al. 2007). In the present calculations, however, the effects of gravity are considered only in the chromosphere, and are not included in the stellar corona and the stream, which is at variance with the model of Orlando et al. (2010). This was done to allow for a more direct comparison of the model results with those of our laboratory experiment where the gravity effects can be considered negligible. The effects of gravity are considered in the stellar chromosphere to have a realistic density stratification there.

Initially, the stellar atmosphere is unperturbed and in magneto-static equilibrium. The vertical profiles of mass density and temperature from the base of the transition region $(T=$ $10^{4} \mathrm{~K}$ ) to the corona are calculated by using the wind model of Orlando et al. (1996), adapted to the conditions of a CTTS. Thus, the stellar atmosphere consists of a hot (maximum temperature $\left.\approx 10^{6} \mathrm{~K}\right)$ and tenuous $\left(n_{\mathrm{H}} \approx 2 \times 10^{8} \mathrm{~cm}^{-3}\right)$ corona that is linked through a steep transition region to an isothermal chromosphere, which we modeled with a temperature of $10^{4} \mathrm{~K}$ and a thickness of $8.5 \times 10^{8} \mathrm{~cm}$.

At the beginning of the simulation, the accretion stream enters into the spatial domain from the upper boundary. As a reference case, we considered a stream with density $n_{\text {str0 }}=$ $10^{11} \mathrm{~cm}^{-3}$ and velocity $u_{\mathrm{str} 0}=-500 \mathrm{~km} \mathrm{~s}^{-1}$ compatible with those derived from the analysis of X-ray spectra of MP Mus (Argiroffi et al. 2007). The stream pressure is determined by the pressure balance across the stream lateral boundary. The unperturbed ambient magnetic field is assumed to be uniform, with a strength $\left|\boldsymbol{B}_{0}\right|=10 \mathrm{G}$, and inclined with respect to the stellar surface with an angle of $\theta=80^{\circ}$. Then, we considered additional simulations in which we alternatively vary the stream
Table 2. Parameters for the MHD models of accretion impacts.

\begin{tabular}{lcccc}
\hline \hline $\begin{array}{l}\text { Model } \\
\text { abbreviation }\end{array}$ & $\begin{array}{c}\left|\boldsymbol{B}_{0}\right| \\
{[\mathrm{G}]}\end{array}$ & $\begin{array}{c}n_{\text {str0 }} \\
{\left[10^{11} \mathrm{~cm}^{-3}\right]}\end{array}$ & $\begin{array}{c}u_{\text {str0 }} \\
{\left[\mathrm{km} \mathrm{s}^{-1}\right]}\end{array}$ & $\theta$ \\
\hline D1e11-B10-A10 & 10 & 1 & 500 & 10 \\
D1e11-B10-A20 & 10 & 1 & 500 & 20 \\
D1e11-B50-A20 & 50 & 1 & 500 & 20 \\
D5e10-B10-A20 & 10 & 0.5 & 500 & 20 \\
D5e10-B50-A20 & 50 & 0.5 & 500 & 20 \\
D5e10-B30-A15 & 30 & 0.5 & 500 & 15 \\
D5e10-B30-A45 & 30 & 0.5 & 500 & 45 \\
\hline
\end{tabular}

density, and the strength and inclination of the stellar magnetic field, around the reference values. Since the stream reproduced in the laboratory experiment has characteristics similar to those of the stream analyzed by Revet et al. (2017), we considered magnetic field strengths that lead to a plasma $\beta$ in the postshock region similar to that found in Revet et al. (2017), ranging between 1 and 100. Table 2 reports a summary of the simulations performed, where $n_{\text {stro }}$ and $u_{\text {str0 }}$ are the initial density and velocity of the stream, respectively, $\left|\boldsymbol{B}_{0}\right|$ is the initial magnetic field strength, and $\theta$ is the inclination angle of the stream with respect to the stellar surface. We note that the values of the magnetic field strength and stream density explored here are in the low range of the values expected in CTTSs (see Table 1); this choice was made because we are interested in studying impacts producing detectable X-ray emission (e.g., Sacco et al. 2010) and in order to have a good spatial resolution of the post-shock region, being the stand-off height of the hot slab generated by the impact proportional to the stream density (e.g., Sacco et al. 2010; Orlando et al. 2010).

We performed the calculations with PLUTO, a modular Godunov-type code for astrophysical plasmas, intended mainly for astrophysical applications and high Mach number flows in multiple spatial dimensions (Mignone et al. 2007). The MHD equations were solved using the MHD module available in PLUTO, configured to compute intercell fluxes with the HartenLax-van Leer Discontinuities (HLLD) approximate Riemann solver, while second order in time was achieved using a RungeKutta scheme. A monotonized central difference limiter for the primitive variables was used. The evolution of the magnetic field was carried out adopting the constrained transport approach (Balsara \& Spicer 1999) that maintains the solenoidal condition $(\nabla \cdot \boldsymbol{B}=0)$ at machine accuracy. The optically thin radiative losses are included in a fractional step formalism (Mignone et al. 2007), which preserves the second time accuracy, as the advection and source steps are accurate at least to the second order. The radiative loss $\Lambda$ values were computed at the temperature of interest using a table look-up/interpolation method. The thermal conduction was treated by adopting the super-timestepping technique (Alexiades et al. 1996), which is very effective in speeding up explicit time-stepping schemes for parabolic problems.

We solved the MHD equations using cartesian coordinates in the plane $(x, z)$. The coordinate system is oriented in such a way that the stellar surface lies on the $x$-axis and the normal to the stellar surface is along the $z$-axis. The stream axis is inclined with respect to the stellar surface and the $x$-axis by the angle $\theta$. The mesh extends between $-2.2 \times 10^{10} \mathrm{~cm}$ and $1.1 \times 10^{10} \mathrm{~cm}$ in the $x$-direction and between $-1.0 \times 10^{9} \mathrm{~cm}$ and $1.4 \times 10^{10} \mathrm{~cm}$ in the $z$-direction. The transition region between the chromosphere 

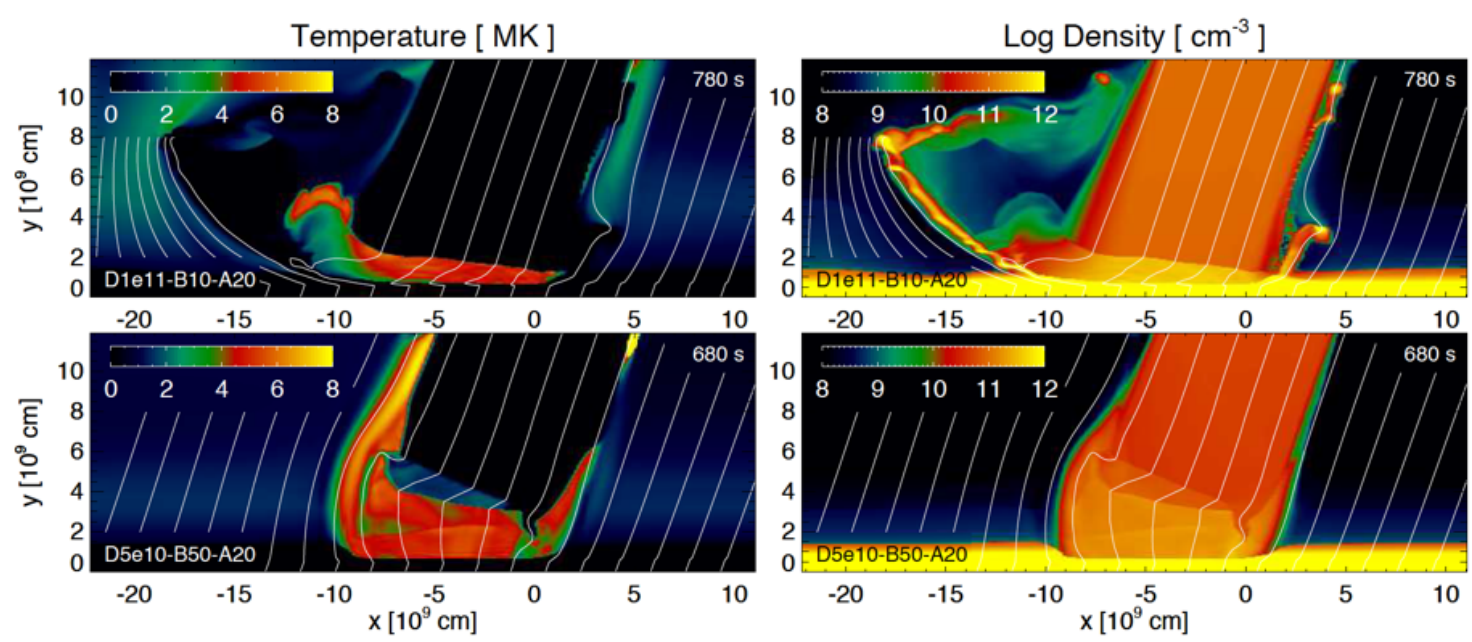

Fig. 10. Spatial distributions of temperature (left panels) and density (right panels in log scale) at the labeled times for a model with high plasma $\beta$ (run D1e11-B10-A20; upper panels) and a model with low plasma $\beta$ (run D5e10-B50-A20; lower panels). The white lines mark magnetic field lines. Movie available online.

and the corona is located at $z=0 \mathrm{~cm}$. The grid along the $x$-axis is uniform and made of $N_{\mathrm{r}}=256$ points with a resolution of $\Delta x \approx 1.3 \times 10^{8} \mathrm{~cm}$; the grid along the $z$-axis is nonuniform with mesh size increasing with $z$. In such a way, the $z$-grid has the highest spatial resolution closer to the stellar chromosphere, thus allowing an accurate description of the steep temperature gradient of the transition region and the evolution of post-shock material, resulting from the impact of the accretion stream with the stellar chromosphere. More specifically, the z-grid is made of $N_{\mathrm{z}}=512$ points and consists of a uniform grid patch with 256 points and a maximum resolution of $\Delta z \approx 10^{7} \mathrm{~cm}$ that covers the chromosphere and the upper stellar atmosphere up to the height of $\approx 1.8 \times 10^{9} \mathrm{~cm}$ and a stretched grid patch for $z>1.8 \times 10^{9} \mathrm{~cm}$ with 256 points and a mesh size increasing with $z$, which leads to a minimum resolution at the upper boundary of $\Delta z \approx 2.8 \times 10^{8} \mathrm{~cm}$.

The boundary conditions are those of free outflow ${ }^{1}$ at $x=$ $-2.2 \times 10^{10} \mathrm{~cm}$ and $x=1.1 \times 10^{10} \mathrm{~cm}$, fixed boundary conditions at $z=-1.0 \times 10^{9} \mathrm{~cm}$ (imposing zero material and heat flux across the boundary), and a constant inflow in the upper boundary at $z=1.4 \times 10^{10} \mathrm{~cm}$.

\subsection{Dynamics of the stream impact}

We found that the evolution of the stream impact is similar to that described in Orlando et al. (2010) when the plasma $\beta$ is high (and the shock-heated plasma is poorly confined by the magnetic field) and to that described in Orlando et al. (2013) when $\beta$ is low (and the post-shock plasma is well confined by the ambient field). The main difference with previous models is that now the stream hits the stellar surface with an incidence angle larger than $0^{\circ}$, and this introduces a marked asymmetry of the density and temperature distributions in the post-shock plasma. Figure 10 shows 2D maps of temperature and density spatial distribution for two models characterized by high or low plasma $\beta$. Movies showing the complete evolution of temperature and density in log scale for all the models listed in Table 2 are provided online. As in previous analogous simulations, the accreting material downflows along the magnetic field lines and hits the stellar chromosphere at $t \sim 250 \mathrm{~s}$. Then the stream gradually

\footnotetext{
Set zero gradients across the boundary.
}

sinks into the chromosphere and it stops sinking when, locally, the thermal pressure of the chromosphere equals the ram pressure of the stream. At this time, a shock develops at the base of the accretion column and propagates upward through the stream, heating the accreting material up to temperatures of a few million degrees (thus contributing to emission mainly in the $\mathrm{X}$-ray band). This newly formed hot slab of plasma is partially rooted in the chromosphere, so that the X-ray-emitting plasma is buried under a column of optically thick material and is partially absorbed (e.g., Reale et al. 2013; Bonito et al. 2014; Revet et al. 2017; Costa et al. 2017; Colombo et al. 2019b). We note that the evolution of the simulated accretion process is shown here over tens of minutes, whereas the scaling discussed in Sect. 2.2 indicated that the laboratory observations (up to $100 \mathrm{~ns}$ ) would correspond to hours in the astrophysical case. However, not only can an accretion column hit the stellar surface for days (e.g., Romanova et al. 2002, 2003; Colombo et al. 2019a), but we also expect that the evolution of the post-shock plasma, on a time scale of hours, will not change significantly in the repetition of alternative rise and collapse of the hot slab (e.g., Orlando et al. 2010, 2013).

Due to the high plasma $\beta$ value in our reference case (upper panels of Fig. 10), the dense hot plasma in the slab causes a pressure-driven flow that ejects part of the accreted material sideways. Due to the stream inclination with respect to the stellar surface, the flow velocity has a component, $v_{\text {perp }}$, perpendicular to the stellar surface. As a result, the effect of impact at the border of the stream is different on each side: the outflow is not symmetric around the stream and is faster and more pronounced in the direction of $v_{\text {perp }}$. This is particularly true in the case of models with high plasma $\beta$ (D1e11-B10-A20, D1e11-B10-A10, D5e10-B10-A20). In these cases, the outflow strongly perturbs the surrounding stellar atmosphere and drags the magnetic field trapped at the head of the escaped material, leading to a continuous increase in the magnetic pressure and field tension there (see upper panels of Fig. 10). In general, due to the presence of the magnetic field, the escaped material does not flow freely, but is redirected upward and (possibly) is pushed back on the stream by the magnetic field tension. In run D5e10-B50-A20, the magnetic field is strong enough to force the escaped material to plunge into the stream and to significantly perturb it (see lower panels of Fig. 10). On the side of the stream in the opposite direction of 

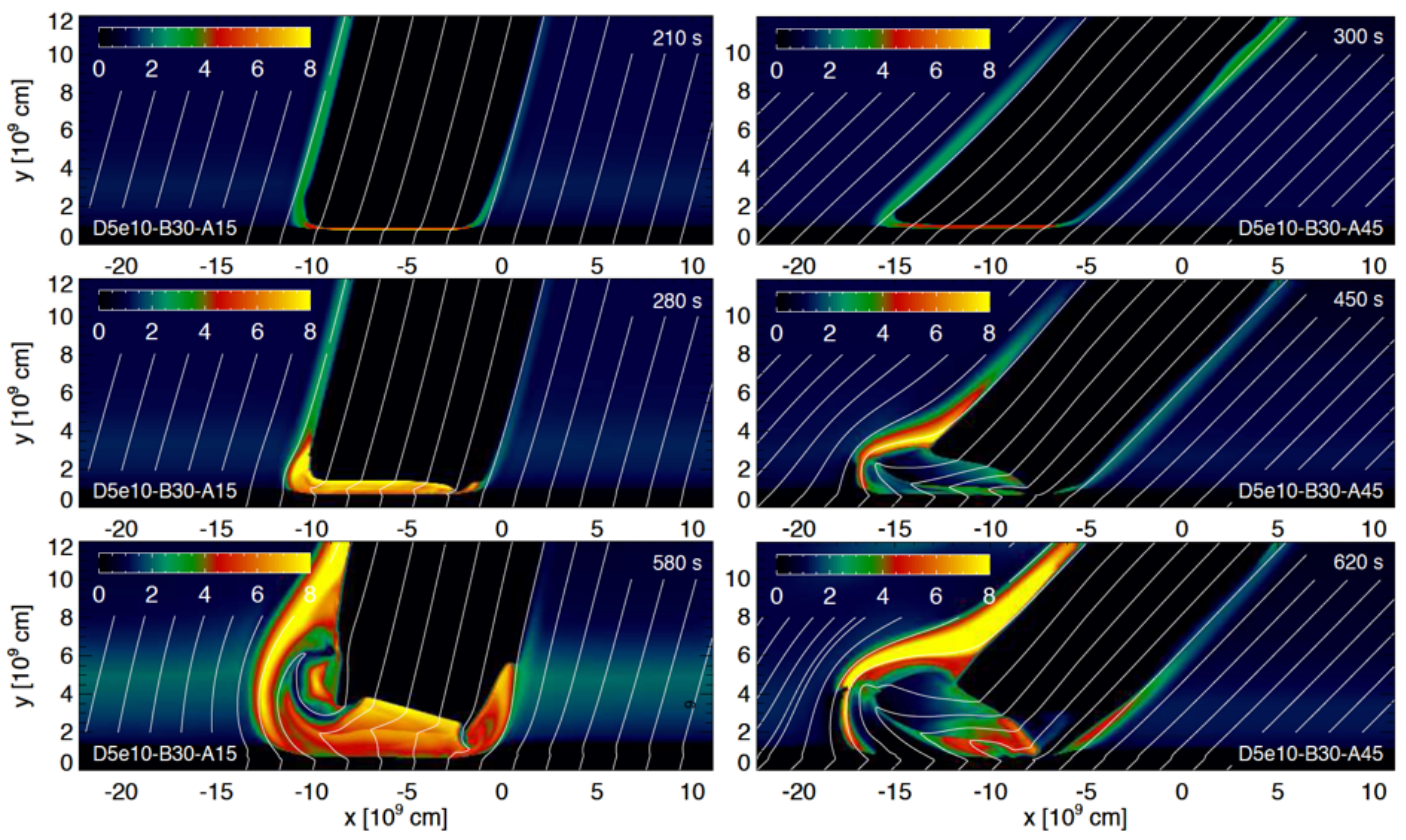

Fig. 11. Evolution of temperature distribution at labeled times for $15^{\circ}$ (run D5e10-B30-A15; on the left) and $45^{\circ}$ (run D5e10-B30-A45; on the right) oblique accretion stream incidence. The white lines mark magnetic field lines. Movie available online.
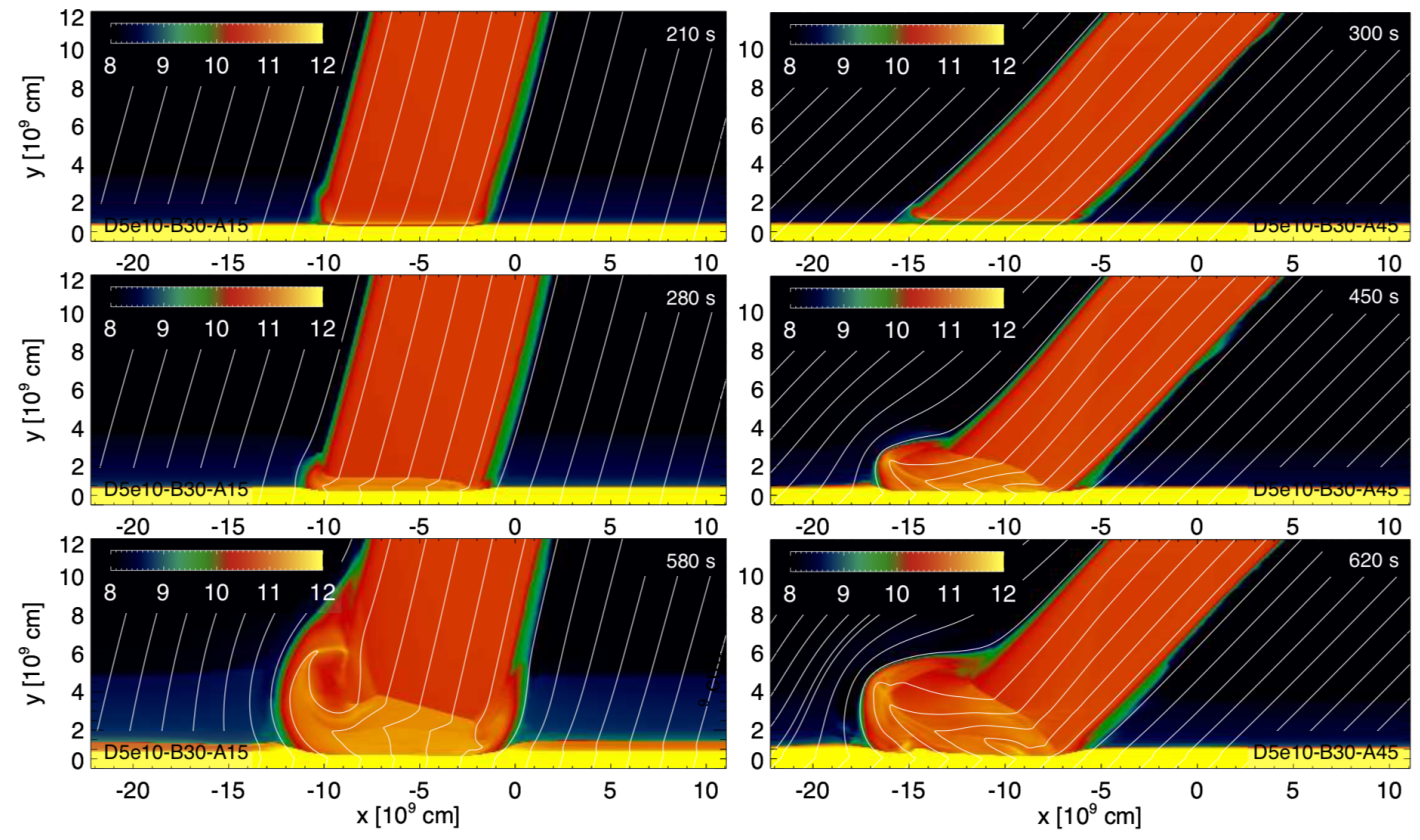

Fig. 12. Spatial distributions of density in $\log$ scale at labeled times for $15^{\circ}$ (run D5e10-B30-A15; on the left) and $45^{\circ}$ (run D5e10-B30-A45; on the right) oblique accretion stream incidence. The white lines mark magnetic field lines.

$v_{\text {perp }}$, the field is strong enough to keep the outflow close to the stream in all the simulations explored. The outflowing plasma accumulates around the accretion column and gradually forms a sheath of turbulent material that envelops the stream.

In all our simulations, the hot slab is thermally unstable due to the radiative cooling. In fact, the continuous accumulation of accreting material at the base of the stream leads to an increase of density in the post-shock plasma, and, as a consequence, to an increase in radiative losses (which depends on the square of density for optically thin plasmas). As a consequence, oscillations of the hot slab (and variability of the outflowing plasma) are induced by radiative cooling. In models with high plasma $\beta$, the variations appear chaotic without an evident periodicity, at least in the time lapse explored here. In models with low plasma $\beta$, quasi-periodic oscillations are observed, although they are strongly perturbed by the sheath of turbulent material around the stream.

Among the models analyzed, those that roughly reproduce the morphology observed in the experiments assume a stream with a density $n_{\text {stro }}=5 \times 10^{10} \mathrm{~cm}^{-3}$ that propagates through a magnetized stellar atmosphere with magnetic field strength $\left|\boldsymbol{B}_{0}\right|=30 \mathrm{G}$. Figures 11 and 12 show the distribution of temperature and density for two incidence angles, namely $15^{\circ}$ and $45^{\circ}$, the same as our experiments in Fig. 2. In the first case, the 
evolution is similar to that described above. In the extreme case of the $45^{\circ}$ oblique accretion stream incidence, the asymmetry is very evident and most of the plasma with a temperature above 1 MK (i.e., the plasma that contributes to X-ray emission arising from the stream impact) is confined on the side of the stream in the direction of $v_{\text {perp }}$.

\subsection{X-ray emission}

We focused on the reference case (run D5e10-B30-A45) described in Fig. 12 and Table 2, to derive detailed information regarding the X-ray emission of the shocked plasma from the accretion stream impacting the star, and regarding the effect of having an oblique stream onto such emission (with respect to the case of a stream normal to the star). To this aim, we performed the synthesis of the X-ray emission that would result from the configuration considered in the reference case, applying the post-processing tool that we developed and successfully used in the investigation of astrophysical shocks produced by streams (e.g., Bonito et al. 2011) and accretion streams (e.g., Bonito et al. 2014). The two cited references not only detail the tool that we used here, but notably show that the spectra synthesized from the numerical models can be directly compared with the astrophysical observations.

We focused our synthesis of the radiation emanating from the modeled accretion impacts on the emission around the O VII and Ne IX triplets in X-rays, in particular exploring the effect of the local absorption on the emission at different wavelengths. The aim is to evaluate how the effects of this local absorption change when the structure of the accretion impact is distorted in the case of a slanted impact. We neglected the contribution to the emission due to the corona and the absorption due to the high-temperature plasma, and we used the Anders \& Grevesse 1989 abundances (as in Bonito et al. 2014). We find that the main contribution to the local absorption is the unperturbed accretion stream itself, as well as the asymmetric lateral ejecta that perturb the ambient, as observed in the laboratory experiment and reproduced by the selected reference model described here. As the ejecta are asymmetric, what is found is that the absorption, and hence the emission, differ depending on the direction from which the emission would be collected from different sides of the accreting column. We synthesized the emission as emanating either from the left or from the right side of the accretion column, which here is modeled in 2D in order to account for the two extremes of minimum and maximum contribution of the local absorption. Indeed, as is clear from Fig. 12, the emission propagating to the right of the figure encounters much less lateral ejecta than that propagating to the left, hence we expect them to be differently affected by local absorption in the plasma surrounding the incoming accretion stream.

We calculated the X-ray emission emanating from each point of the computational domain, both neglecting local absorption and taking its effect into account. For the latter, we weighted the emission by absorption induced by the plasma located between any point of the simulation grid and an observer that would be located either on the left or on the right side of the stream. Part of the emission (at the base of the shock) is totally absorbed due to the sinking of the shock into the chromosphere. Figure 13 shows the total emission emanating from the plasma, as a function of the height above the star surface. The emission is shown for three cases: without considering any absorption in the plasma (black continuous line), and considering absorption, but as seen either from the right (green dashed line) or the left (red doted line) of the column. The last two cases represent the minimum

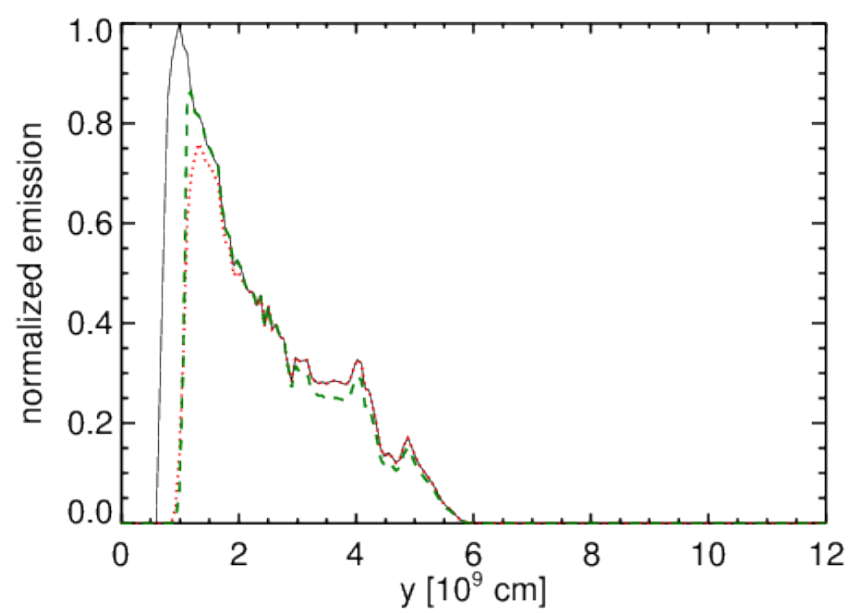

Fig. 13. Simulated X-ray emission emanating from accretion column modeled in run D5e10-B30-A45, as a function of the height above the star surface $(z)$, for three cases: without any local absorption effect (black continuous line), and with the local absorption integrating the emission from the right (green dashed line) and from the left (red dotted line) side of the accretion stream.

and maximum effect of the local absorption due to the accretion stream itself as well as due to the asymmetric lateral perturbations detected in both the laboratory experiment and the numerical model. We naturally observe in Fig. 13 a reduction in the peak of the emission when considering absorption, with that reduction being different depending on whether the accretion column is viewed from the right or the left. Obviously, this is due to the asymmetric nature of the lateral ejecta. The peak corresponding to the bright blob located at the height of $4 \times 10^{9} \mathrm{~cm}$ is almost identical in the unabsorbed case and in the absorbed case synthesized from the left side of the accretion stream (compare second peak in Fig. 13 in black continuous line and red dotted line), while the main peak (the bright region at the base of the shock, but not sinked in the chromosphere) is strongly reduced due to the local absorption contribution of the lateral asymmetrical material.

In the absorbed case synthesized from the right side of the accretion stream (green dashed line in Fig. 13), both the peaks of intensity (the one at the base, and the one located at $4 \times 10^{9} \mathrm{~cm}$ ) are strongly suppressed with respect to the unabsorbed case, but the main peak is higher with respect to the absorbed case observed from the left side of the shock (red dotted line in Fig. 13). In this case, the contribution to the emission due to the bright region at the base of the shock (not sunk into the chromosphere) is less absorbed than in the previous case, as an effect of the asymmetric lateral structure formed as a consequence of the slanted accretion stream. The second peak of emission higher with respect to the stellar surface (at approximate $4 \times 10^{9} \mathrm{~cm}$, see Fig. 13) is more absorbed due to the local absorption of the accretion stream itself. The reduction of the main peak (located at approximately $1.5 \times 10^{9} \mathrm{~cm}$, see Fig. 13) with respect to the case without the local absorption taken into account corresponds to approx. $14 \%$ in the absorbed case observed from the right side of the stream (green dashed line in Fig. 13) and to approx. 24\% in the absorbed case observed from the left side of the stream (red dotted line in Fig. 13), due to the asymmetrical structures formed in the slanted accretion stream. This result highlights the role of the local absorption and the importance of the lateral structures formed as a consequence of the inclination of the accretion stream with respect to the stellar surface. 
We also simulated the spectra, in particular in the spectral range of the relevant triplets used for X-ray diagnostic (O VII and Ne IX). In both cases, we observe a reduction of the peak of the emission when the local absorption is taken into account, which is again different depending on the direction from which the observation is made.

\section{Discussion and conclusions}

On the basis of the experimental data, we can deduce: (1) a lack of confinement of plasma around the accretion column with the increasing of the tilt of the incoming stream, which is confirmed by interferometry, SOP, and VSG; (2) the lateral escaping of the accreting plasma and increasing asymmetry of the accretion column, which is confirmed by interferometry and SOP; (3) a reduction in the temperature of the impacting plasma, which confirmed by VSG.

All of this is well supported by the astrophysical simulations presented above and can be summarized in terms of plasmamagnetic field interaction. Indeed, at impact, in the case of a stream impacting the obstacle normally, the laterally ejected plasma along the obstacle surface encounters a perpendicular magnetic field, capable of stopping that lateral ejecta and refocusing it at the edges of the incoming stream (Revet et al. 2017). However, when increasing the inclination of the obstacle, the ejected material also encounters an increasingly oblique magnetic field, having an inferior capability to stop the flow. All this leads to lesser plasma confinement, reduced heating, and increased plasma propagation along the obstacle surface in an increasingly asymmetric configuration.

Overall, both the experiments and the MHD simulations predict that the stellar atmosphere in the immediate surroundings of an impact region of an accretion stream can be heavily perturbed by the impact. According to simulations and experiments, the perturbation increases with the incidence angle, especially on the side of the stream in the direction of $v_{\text {perp }}$ where a plasma motion parallel to the stellar surface can be generated at the base of the stream. Depending on the plasma $\beta$, the magnetic field can be significantly advected by these outflows, and the effect increases for higher incidence angles of the stream. In these cases, the conditions for ideal MHD may break down. Several authors have shown that perturbations traveling through the solar atmosphere and interacting with magnetic field lines can induce strong instabilities that lead to reconnective phenomena (e.g. Isobe et al. 2007; Li \& Zhang 2012; Jackiewicz \& Balasubramaniam 2013; Jess et al. 2015, and references therein). Thus, we expect that perturbations induced by the lateral flows may incite magnetic reconnection in proximity to the impact region, leading to a local release of the stored energy from the magnetic field that may heat up the surrounding stellar atmosphere (e.g., Reale et al. 2016). These effects are not described by our models as they do not include resistivity effects. From the experiments, we do not see any indication of heating release, but we have to underline the fact that the X-ray emission we recorded is dominated by the dense on-axis plasma, when possible heating would laterally affect ejected low-density plasma, which would then contribute little to the overall emission.

In addition to possible magnetic reconnection events that may occur in proximity to impact regions, we expect stream impacts with a significant incidence angle to easily trigger MHD waves which propagate in the chromosphere, and which again may perturb the structure of the chromosphere, and, possibly, of the overlying corona. In the Sun, MHD waves can be initiated as a consequence of several events such as eruptive flares triggering oscillatory phenomena (e.g., Verwichte et al. 2004; De Moortel \& Brady 2007; Van Doorsselaere et al. 2007, 2009: Srivastava \& Goossens 2013; Shen et al. 2014; Jess et al. 2015), or large-scale coronal streamers following the impact of a rapidly propagating coronal mass ejection (e.g., Chen et al. 2010, 2011). More recently, solar observations have recorded impacts of falling fragments after the eruption of a filament in a flare that has physical characteristics (infalling speed and density) close to those inferred for accretion flows on young accreting stars (Reale et al. 2013). The analysis of observations and their comparison with hydrodynamic models describing these impacts reveal a strong perturbation of the solar atmosphere (chromosphere and corona) and the triggering of oscillatory motion (Reale et al. 2014; Petralia et al. 2016, 2017). In our study, the generation of waves is expected to be the largest on the side of the stream in the direction of $v_{\text {perp }}$, where the chromosphere is more perturbed by the stream impact.

The possibility that stream impacts may produce a significant perturbation of the stellar atmosphere has implications for the evidence that the observed coronal activity is apparently influenced by accretion (but it is not clear why and how: Neuhaeuser et al. 1995; Drake et al. 2009). Some authors proposed that the coronal activity is modulated by mass accretion through the suppression, disruption, or absorption of the coronal magnetic activity (e.g., Flaccomio et al. 2003; Stassun et al. 2004; Preibisch et al. 2005; Jardine et al. 2006; Gregory et al. 2007). Others proposed that the accretion may enhance the coronal activity around impact regions due to heating of the surrounding stellar atmosphere to soft X-ray-emitting temperatures (e.g., Brickhouse et al. 2010; Dupree et al. 2012). Our analysis suggests that accretion impacts with an incidence angle may produce significant outflows at the base of accretion streams; we propose that these outflows may disrupt or suppress coronal magnetic activity around impact regions. In the case of magnetic reconnection events in proximity of impact regions, we expect that the coronal activity may be enhanced. The perturbation of the stellar atmosphere may also contribute to the stellar outflow as suggested by Cranmer (2008) through a model of accretiondriven winds in CTTSs (see also, Cranmer 2009). According to this model, the mass-loss rates observed in CTTSs can be explained if, in addition to the convection-driven MHD turbulence that dominates in solar-like stars, a source of wave energy driven by stream impacts onto the stellar surface is present. Future modeling studies including resistive effects and an accurate description of the density and temperature structure of the stellar atmosphere (from the photosphere to the chromosphere and to the corona) and of the stellar magnetic field in proximity of impact regions may offer additional insight into the reaction of the stellar atmosphere to accretion impacts and shed light on the connection between mass accretion rates and level of corona activity.

Acknowledgements. This work was supported by funding from the European Research Council (ERC) under the European Unions Horizon 2020 research and innovation program (Grant Agreement No. 787539), as well as ANR Blanc Grant n 12-BS09-025-01 SILAMPA (France) and by the Russian Foundation for Basic Research (RFBR) foundation in the frame of projects No. 18-29-21018, No. 1802-00850, and No. 19-32-60008. The JIHT RAS team's work is supported by the grant No. 13.1902.21.0035 in the form of a subsidy from Ministry of Science and Higher Education of the Russian Federation. This work was partly done within the LABEX Plas@Par, the DIM ACAV funded by the Region Ilede-France, and supported by Grant No. 11-IDEX- 0004-02 from ANR (France). Part of the experimental system is covered by a patent (n 1000183285, 2013, INPI-France). The research leading to these results is supported by Extreme Light Infrastructure Nuclear Physics (ELI-NP) Phase II, a project co-financed by 
the Romanian Government and European Union through the European Regional Development Fund. The PLUTO code is developed at the Turin Astronomical Observatory (Italy) in collaboration with the Department of General Physics of Turin University (Italy) and the SCAI Department of CINECA (Italy). The astrophysical simulations were carried out at the SCAN (Sistema di Calcolo per l'Astrofisica Numerica) facility for high performance computing at INAF Osservatorio Astronomico di Palermo (Italy).

\section{References}

Albertazzi, B., Ciardi, A., Nakatsutsumi, M., et al. 2014, Science, 346, 325

Alexiades, V., Amiez, G., \& Gremaud, P. A. 1996, Commun. Numer. Methods Eng., 12, 31

Anders, E., \& Grevesse, N. 1989, Geochim. Cosmochim. Acta, 53, 197

Argiroffi, C., Maggio, A., \& Peres, G. 2007, A\&A, 465, L5

Argiroffi, C., Drake, J. J., Bonito, R., et al. 2017, A\&A, 607, A14

Balsara, D. S., \& Spicer, D. S. 1999, J. Comput. Phys., 149, 270

Bonito, R., Orlando, S., Miceli, M., et al. 2011, ApJ, 737, 54

Bonito, R., Orlando, S., Argiroffi, C., et al. 2014, ApJ, 795, L34

Brickhouse, N. S., Cranmer, S. R., Dupree, A. K., Luna, G. J. M., \& Wolk, S 2010, ApJ, 710, 1835

Caratti o Garatti, S. R., Stecklum, B., Garcia Lopez, R., et al. 2017, Nat. Phys., 13,276

Chen, Y., Song, H. Q., Li, B., et al. 2010, ApJ, 714, 644

Chen, Y., Feng, S. W., Li, B., et al. 2011, ApJ, 728, 147

Colombo, S., Orlando, S., Peres, G., et al. 2019a, A\&A, 624, A50

Colombo, S., Ibgui, L., Orlando, S., et al. 2019b, A\&A, 629, L9

Costa, G., Orlando, S., Peres, G., Argiroffi, C., \& Bonito, R. 2017, A\&A, 597, A1

Cowie, L. L., \& McKee, C. F. 1977, ApJ, 211, 135

Cranmer, S. R. 2008, ApJ, 689, 316

Cranmer, S. R. 2009, ApJ, 706, 824

Curran, R. L., Argiroffi, C., Sacco, G. G., et al. 2011, A\&A, 526, A104

Dalton, W. W., \& Balbus, S. A. 1993, ApJ, 404, 625

De Moortel, I., \& Brady, C. S. 2007, ApJ, 664, 1210

Drake, J. J., Ercolano, B., Flaccomio, E., \& Micela, G. 2009, ApJ, 699, L35

Dupree, A. K., Brickhouse, N. S., Cranmer, S. R., et al. 2012, ApJ, 750, 73

Espinosa, G., Rodriguez, R., Gil, J. M., et al. 2017, Phys. Rev. E, 95, 033201

Faenov, A. Y., Pikuz, S. A., Erko, A. I., et al. 1994, Phys. Scr., 50, 333

Filippov, E. D., Skobelev, I. Y., Revet, R., et al. 2019, Matter Radiat. Extremes, 4, 064402

Flaccomio, E., Damiani, F., Micela, G., et al. 2003, ApJ, 582, 398

Gregory, S. G., Wood, K., \& Jardine, M. 2007, MNRAS, 379, L35

Hartmann, L., Herczeg, G., \& Calvet, N. 2016, ARA\&A, 54, 135

Haugh, M. J., Lee, J., Romano, E., \& Schneider, M. 2013, in Target Diagnostics Physics and Engineering for Inertial Confinement Fusion II, eds. P. M. Bell, \& G. P. Grim , International Society for Optics and Photonics (SPIE), 8850, 45

Higginson, D., Revet, G., Khiar, B., et al. 2017a, High Energy Density Phys., 23, 48

Higginson, D. P., Khiar, B., Revet, G., et al. 2017b, Phys. Rev. Lett., 119, 255002
Isobe, H., Tripathi, D., Asai, A., \& Jain, R. 2007, Sol. Phys., 246, 89 Jackiewicz, J., \& Balasubramaniam, K. S. 2013, ApJ, 765, 15 Jardine, M., Collier Cameron, A., Donati, J.-F., Gregory, S. G., \& Wood, K. 2006, MNRAS, 367, 917

Jess, D. B., Morton, R. J., Verth, G., et al. 2015, Space Sci. Rev., 190, 103

Kashyap, V., \& Drake, J. J. 2000, Bull. Astron. Soc. India, 28, 475

Kita, T., Harada, T., Nakano, N., \& Kuroda, H. 1983, Appl. Opt., 22, 512

Li, T., \& Zhang, J. 2012, ApJ, 760, L10

Matsakos, T., Chièze, J. P., Stehlé, C., et al. 2013, A\&A, 557, A69

Mignone, A., Bodo, G., Massaglia, S., et al. 2007, ApJS, 170, 228

Neuhaeuser, R., Sterzik, M. F., Schmitt, J. H. M. M., Wichmann, R., \& Krautter, J. 1995, A\&A, 297, 391

Orlando, S., Lou, Y.-Q., Rosner, R., \& Peres, G. 1996, J. Geophys. Res., 101, 24443

Orlando, S., Bocchino, F., Reale, F., Peres, G., \& Pagano, P. 2008, ApJ, 678, 274

Orlando, S., Sacco, G. G., Argiroffi, C., et al. 2010, A\&A, 510, A71

Orlando, S., Reale, F., Peres, G., \& Mignone, A. 2011, MNRAS, 415, 3380

Orlando, S., Bonito, R., Argiroffi, C., et al. 2013, A\&A, 559, A127

Park, J., Brown, G. V., Schneider, M. B., et al. 2010, Rev. Sci. Instrum., 81, 10 E319

Petralia, A., Reale, F., Orlando, S., \& Testa, P. 2016, ApJ, 832, 2

Petralia, A., Reale, F., \& Orlando, S. 2017, A\&A, 598, L8

Preibisch, T., Kim, Y.-C., Favata, F., et al. 2005, ApJS, 160, 401

Reale, F., Orlando, S., Testa, P., et al. 2013, Science, 341, 251

Reale, F., Orlando, S., Testa, P., Landi, E., \& Schrijver, C. J. 2014, ApJ, 797, L5

Reale, F., Orlando, S., Guarrasi, M., et al. 2016, ApJ, 830, 21

Revet, G., Chen, S. N., Bonito, R., et al. 2017, Science Advances, 3, e1700982

Revet, G., Khiar, B., Béard, J., et al. 2019, High Energy Density Phys., 33, 100711

Rodriguez, R., Florido, R., Gil, J. M., et al. 2008, Laser Part Beams, 26, 433

Romanova, M. M., Ustyugova, G. V., Koldoba, A. V., \& Lovelace, R. V. E. 2002, ApJ, 578, 420

Romanova, M. M., Ustyugova, G. V., Koldoba, A. V., Wick, J. V., \& Lovelace, R. V. E. 2003, ApJ, 595, 1009

Ryutov, D. D. 2018, Phys. Plasmas, 25, 100501

Ryutov, D. D., Drake, R. P., \& Remington, B. A. 2000, ApJS, 127, 465

Sacco, G. G., Orlando, S., Argiroffi, C., et al. 2010, A\&A, 522, A55

Scaringi, S., Maccarone, T. J., Körding, E., et al. 2015, Sci. Adv., 1

Shen, Y., Ichimoto, K., Ishii, T. T., et al. 2014, ApJ, 786, 151

Smith, R. K., Brickhouse, N. S., Liedahl, D. A., \& Raymond, J. C. 2001, ApJ, 556, L91

Spitzer, L. 1962, Physics of Fully Ionized Gases (New York: Interscience)

Srivastava, A. K., \& Goossens, M. 2013, ApJ, 777, 17

Stassun, K. G., Ardila, D. R., Barsony, M., Basri, G., \& Mathieu, R. D. 2004, AJ, 127, 3537

Telleschi, A., Güdel, M., Briggs, K. R., Audard, M., \& Scelsi, L. 2007, A\&A, 468, 443

Van Doorsselaere, T., Nakariakov, V. M., \& Verwichte, E. 2007, A\&A, 473, 959 van Doorsselaere, T., Birtill, D. C. C., \& Evans, G. R. 2009, A\&A, 508, 1485

Verwichte, E., Nakariakov, V. M., Ofman, L., \& Deluca, E. E. 2004, Sol. Phys., 223, 77

Zou, J. P., Blanc, C. L., Audebert, P., et al. 2008, J. Phys.: Conf. Ser., 112, 032021 